February 1, 2008

hep-th/9711143

\title{
Kähler Potential and Higher Derivative Terms from M Theory Fivebrane
}

\author{
Jan de Boer, Kentaro Hori, Hirosi Ooguri and Yaron Oz \\ Department of Physics, University of California at Berkeley \\ 366 Le Conte Hall, Berkeley, CA 94720-7300, U.S.A. \\ and \\ Theoretical Physics Group, Mail Stop 50A-5101 \\ Ernest Orlando Lawrence Berkeley National Laboratory \\ Berkeley, CA 94720, U.S.A.
}

\begin{abstract}
The construction of four dimensional supersymmetric gauge theories via the fivebrane of $\mathrm{M}$ theory wrapped around a Riemann surface has been successfully applied to the computation of holomorphic quantities of field theory. In this paper we compute non-holomorphic quantities in the eleven dimensional supergravity limit of $M$ theory. While the Kähler potential on the Coulomb of $N=2$ theories is correctly reproduced, higher derivative terms in the $N=2$ effective action differ from what is expected for the four dimensional gauge theory. For the Kähler potential of $N=1$ theories at an abelian Coulomb phase, the result again differs from what is expected for the four-dimensional gauge theory. Using a gravitational back reaction method for the fivebrane we compute the metric on the Higgs branch of $N=2$ gauge theories. Here we find an agreement with the results expected for the gauge theories. A similar computation of the metric on $N=1$ Higgs branches yields information on the complex structure associated with the flavor rotation in one case and the classical metric in another. We discuss what four-dimensional field theory quantities can be computed via the fivebrane in the supergravity limit of $M$ theory.
\end{abstract}




\section{Introduction}

Many gauge field theory results in various dimensions have been obtained in the last year by realizing them on the worldvolume of branes. Another method applied to the study of gauge theories is geometric engineering [1-3]. In this paper we will be interested in studying four dimensional gauge theories using the first method.

Webs of intersecting branes as a tool for studying gauge theories with reduced number of supersymmetries have been introduced in [4]. Such a web of intersecting branes of Type IIA string theory describing $N=2$ gauge theories in four dimensions can be realized by a single fivebrane of $\mathrm{M}$ theory wrapping a Riemann surface [3,5]. The Riemann surface is the Seiberg-Witten curve [6] and therefore the fivebrane configuration encodes the structure of the moduli space of vacua. Similar webs of intersecting branes of Type IIA string theory describing $N=1$ gauge theories in four dimensions $[7,8]$ can be realized by a single fivebrane of $\mathrm{M}$ theory wrapping a Riemann surface. The fivebrane configurations corresponding to these $N=1$ supersymmetric gauge theories encode the information about the $N=1$ moduli spaces of vacua [9-20]

So far the fivebrane construction has been successfully applied to the computation of holomorphic (or rather BPS) quantities of the four dimensional supersymmetric gauge theory. Of particular importance are the non-holomorphic quantities such as higher derivative terms in $N=2$ theories and the Kähler potential of $N=1$ supersymmetric gauge theories. It is very difficult to compute these objects by field theory techniques in particular in regions of strong coupling.

There are two questions to be asked: Can we compute these non holomorphic quantities using the fivebrane and do the results agree with what we expect for the gauge theories in four dimensions? As is well known, the theory on the M theory fivebrane is a $(0,2)$ theory in six dimensions. When wrapping a Riemann surface $\Sigma$ the four dimensional theory on $\mathbf{R}^{4}$ has two scales: The radius of the eleventh dimension $R$ and the typical scale of the brane configuration $L_{\text {brane }}$. There are two corresponding Kaluza-Klein modes with masses $1 / R$ and $1 / L_{\text {brane }}$. The four dimensional gauge field theories that we are interested in have one scale $\Lambda$. In order to correctly obtain these four dimensional theories we have to find the region of values of the parameters $R, L_{\text {brane }}$ where the wrapped fivebrane theory

and the gauge theory agree. This in particular requires a decoupling of the Kaluza-Klein modes.

Holomorphic quantities of field theory are not sensitive to the region of parameters $R, L_{\text {brane }}$ where they are computed. In particular they can also be computed in the eleven 
dimensional supergravity limit of $\mathrm{M}$ theory even though the $1 / R$ Kaluza-Klein modes are light. It is not clear whether this holds also for the computation of the non-holomorphic quantities. The aim of this paper is to answer this question. We will use the fivebrane of eleven dimensional supergravity in order to compute non holomorphic quantities and compare with what we expect from field theory.

The paper is organized as follows:

Section 2 includes a brief discussion of the eleven dimensional supergravity fivebrane action which will be needed for the computations. It also contains a derivation of the formula for the Kähler potential of the four dimensional theory obtained via wrapping the fivebrane on a Riemann surface. In section 3 we use this formula to show that the Kähler potential on the Coulomb branch of $N=2$ theories is correctly reproduced. In section 4 we compute the four derivative term in the $N=2$ effective action which is a non holomorphic quantity. We find an explicit dependence on the radius $R$ of the eleventh dimension. We compare the result with what we expect for the four dimensional gauge field theory and show that the results disagree for any value of $R$. In section 5 we use the fivebrane to compute the Kähler potential of $N=1$ gauge theories in an abelian Coulomb phase. We compare the brane result with what we expect for the four dimensional gauge field theory. Although the effective coupling is correctly reproduced the Kähler potential again disagrees with what we expect for the field theory. In section 6 we study the Higgs branch of $N=2$ theories. In this case the fivebrane worldvolume consists of several disjoint components whose motions parametrize the Higgs branch. We compute the effect of the gravitational force on each component due to the other components and obtain correctly the metric of the $N=2$ Higgs branch, up to possible corrections due to membranes wrapping supersymmetric 3 -cycles. In section 7 we study the metric on the $N=1$ Higgs branch using the same method. We consider $N_{f}=N_{c}=2$ SQCD with and without a heavy adjoint chiral multiplet. In the case with heavy adjoint, we find an indication that the complex structure is correctly reproduced and the result leads to a proposal on the precise relation between the flavor rotation and the motion of a finite component of the fivebrane. In the case without adjoint, the computation only captures classical features of the metric on the Higgs branch. Section 8 is devoted to a discussion of the results.

\section{Preliminaries}

At low energies, $M$ theory is described by eleven dimensional supergravity, and the classical action describing the $M$ theory fivebrane has been determined in [21-23]. We 
will only consider the bosonic part of the fivebrane action, the rest of the fivebrane action is determined by supersymmetry. The world-volume fields of the fivebrane consist of fields $X^{\mu}\left(x^{a}\right)$, where $\mu=0, \ldots, 10$ and $a=0, \ldots, 5$, describing the embedding of the sixdimensional world-volume in eleven-dimensional spacetime, and of a self-dual two-form $B_{a b}$. In addition, the fivebrane action will depend on the eleven-dimensional background fields, which are the metric $G_{\mu \nu}$ and the three-form $C_{\mu \nu \rho}^{(3)}$. The bosonic part of the action expanded up to second order in $B_{a b}$ reads, up to a Wess-Zumino term,

$$
S=\frac{1}{\ell_{11}^{6}} \int \mathrm{d}^{6} x \sqrt{-g}+\int \mathrm{d}^{6} x\left|d B-C^{(3)}\right|^{2},
$$

where $g=\operatorname{det}\left(g_{a b}\right)$ and $g_{a b}$ is the induced metric

$$
g_{a b}=G_{\mu \nu} \partial_{a} X^{\mu} \partial_{b} X^{\nu}
$$

The self-duality constraint for the two-form is that $d B-C^{(3)}$ should be a self-dual threeform with respect to the induced metric $g_{a b}$. In fact, (2.1) does not completely define the theory of a self-dual three-form. For that, one has to add an additional term involving an auxiliary scalar as in [21] which yields in a specific gauge the formulation of [22]. Alternatively, one can extract from the partition function of (2.1) the piece relevant for the self-dual three form as in [24].

The theories that we consider in this paper are obtained from fivebranes of the form $\mathbf{R}^{4} \times \Sigma$ embedded into a spacetime of the form $\mathbf{R}^{4} \times M^{7}$. Here, the two $\mathbf{R}^{4}$ 's are to be identified with each other, and $\Sigma$ is a Riemann surface embedded in $M^{7}$. By performing a Kaluza-Klein reduction of the fivebrane theory on the Riemann surface $\Sigma$ we obtain a four-dimensional theory. As we discuss later, this reduction can be quite subtle, especially in the case where the Riemann surface is non-compact. The various bosonic fields in the four-dimensional theory arise as follows. First, in general there will be family of Riemann surfaces $\Sigma\left(u_{\alpha}\right)$ depending on moduli $u_{\alpha}$. These moduli become scalar fields in the fourdimensional theory. Second, if the Riemann surface has components of finite volume, additional scalar fields arise by taking $B_{a b}$ proportional to the volume form of one of these components. Finally, if the Riemann surface has genus $g$ greater than zero there will be $g U(1)$ vector fields coming from the decomposition of $B_{a b}$ in terms of the harmonic one-forms on $\Sigma$. Although the full fivebrane action is rather complicated, the two terms given in (2.1) will be sufficient for our purposes, as these are the only ones contributing to the terms involving two derivatives in the four dimensional theory. In the cases where we consider higher derivative terms $B$ and $C^{(3)}$ do not contribute, and (2.1) will again be all we need. 
To determine the action of the four-dimensional theory, we need to consider fivebranes of the form $\mathbf{R}^{4} \times \Sigma$ where $\Sigma$ is allowed to vary over $\mathbf{R}^{4}$. More precisely, we assume that the metric on spacetime $\mathbf{R}^{4} \times M_{7}$ is of the form

$$
d s^{2}=f\left(X^{i}\right) \eta_{m n} d X^{m} d X^{n}+G_{i j} d X^{i} d X^{j}
$$

with $m=0, \ldots, 3$ and $i=4, \ldots, 10$, and consider fivebranes with world-volume coordinates $x^{m}, z, \bar{z}$ whose embeddings are of the form

$$
\begin{aligned}
& X^{m}=x^{m}, \quad m=0, \ldots, 3 \\
& X^{i}=X^{i}\left(z, \bar{z}, u_{\alpha}\left(x^{m}\right)\right), \quad i=4, \ldots, 10 .
\end{aligned}
$$

Here, $z, \bar{z}$ are arbitrary coordinates on the Riemann surface $\Sigma\left(u_{\alpha}\right)$. As the fivebrane action is invariant under world-volume diffeomorphisms, we can always choose $z$ and $\bar{z}$ in such a way that the induced metric on the Riemann surface is conformal, i.e. $g_{z z}=g_{\bar{z} \bar{z}}=0$. As this will simplify things considerably, we will from now on always assume this to be the case.

The first term in the fivebrane action (2.1), when evaluated for (2.4), yields

$$
S=\frac{1}{\ell_{11}^{6}} \int d^{4} x d^{2} z g_{z \bar{z}} \sqrt{-\operatorname{det}\left(f\left(X^{i}\right) \eta_{m n}+L_{m n}\right)}
$$

where

$$
L_{m n}=\frac{\partial u_{\alpha}}{\partial x^{m}} \frac{\partial u_{\beta}}{\partial x^{n}}\left(g_{\alpha \beta}-g_{\alpha z} \frac{1}{g_{z \bar{z}}} g_{\beta \bar{z}}-g_{\beta z} \frac{1}{g_{z \bar{z}}} g_{\alpha \bar{z}}\right)
$$

and

$$
g_{\alpha \beta}=\frac{\partial X^{i}}{\partial u_{\alpha}} G_{i j} \frac{\partial X^{j}}{\partial u_{\beta}}, \quad g_{\alpha z}=\frac{\partial X^{i}}{\partial u_{\alpha}} G_{i j} \frac{\partial X^{j}}{\partial z} .
$$

In particular, the kinetic term for the scalars $u_{\alpha}$ reads

$$
S_{k i n}=\frac{1}{\ell_{11}^{6}} \int d^{4} x d^{2} z g_{z \bar{z}} f\left(X^{i}\right) \operatorname{Tr}(L) .
$$

A simplification arises when spacetime is of the form $\mathbf{R}^{4} \times M_{6} \times \mathbf{R}$, where $M_{6}$ is a Kähler manifold, and when $\Sigma$ is holomorphically embedded in $M_{6}$ and depends holomorphically on the moduli $u_{\alpha}$ (which should therefore be complex). We also take $f\left(X^{i}\right)=1$, so that the metric reads

$$
d s^{2}=\eta_{m n} d X^{m} d X^{n}+2 G_{i \bar{j}} d X^{i} d X^{\bar{j}}+\left(d X^{10}\right)^{2} .
$$

In this case the fivebrane configuration preserves $N=1$ supersymmetry in four dimensions and the kinetic term for the scalars should be given by a Kähler metric. The kinetic term is given by

$$
S=\int d^{4} x \partial_{m} u_{\alpha} \partial^{m} u_{\bar{\beta}} K_{\alpha \bar{\beta}}
$$


with

$$
K_{\alpha \bar{\beta}}=\frac{2}{\ell_{11}^{6}} \int_{\Sigma} d^{2} z\left(g_{z \bar{z}} g_{\alpha \bar{\beta}}-g_{z \bar{\beta}} g_{\alpha \bar{z}}\right) .
$$

This metric is indeed a Kähler metric. In fact, it is easy to give an expression for the corresponding Kähler potential. If $K_{\text {spacetime }}\left(X^{i}, X^{\bar{i}}\right)$ is the Kähler potential for the spacetime metric $G_{i \bar{j}}$, i.e. $G_{i \bar{j}}=\partial_{i} \partial_{\bar{j}} K_{\text {spacetime }}$, then

$$
K_{\text {fieldtheory }}=\frac{1}{2 \ell_{11}^{6}} \int_{\Sigma} d^{2} z g_{z \bar{z}} K_{\text {spacetime }}\left(X^{i}\left(z, u_{\alpha}\right), X^{\bar{i}}\left(\bar{z}, u_{\bar{\alpha}}\right)\right) .
$$

In words, the Kähler potential of the $N=1$ field theory in four dimensions is the integral of the spacetime Kähler potential over $\Sigma$ with its induced metric.

The second term in (2.1) can also contribute to the kinetic terms for the scalars and gauge fields in four dimensions. If the Riemann surface has genus $g$, the decomposition of $B_{a b}$ in terms of the harmonic one-forms on the Riemann surface yields $g U(1)$ vector fields in four dimensions. The gauge coupling for these vector fields is given by the imaginary part of the period matrix of the Riemann surface, $S \sim \int\left(\operatorname{Im}\left(\tau_{i j}\right) F_{i} \wedge * F_{j}\right)$ [5]. The contribution of the second term in (2.1) to the kinetic term of the scalars is more complicated and depends on the precise situation. We will only make a few general remarks and postpone a more detailed discussion until we meet concrete examples where this second term is relevant.

The second term in (2.1) is invariant under $\delta B=C^{(2)}, \delta C^{(3)}=d C^{(2)}$. The twoform $B$ can give rise to additional scalar and vector fields in four dimensions. Given some background three-from $C^{(3)}$, we should according to the principle of Kaluza-Klein reduction choose $B$ in such a way that $\int\left|d B-C^{(3)}\right|^{2}$ gives rise to kinetic terms for the four-dimensional fields only, without a mass term, as we are only interested in the fourdimensional fields that are massless. In addition, we have to worry about the gauge invariance and the self-duality condition. Thus, in determining $B$ we should impose two additional constraints. First, $d B-C^{(3)}$ should be a self-dual three-form, and second, certain gauge fixing conditions should be satisfied. As gauge fixing conditions we will use the Lorentz gauges $d *\left(B-B_{0}\right)$ and $d *\left(C^{(3)}-C_{0}^{(3)}\right)=0$, for some fixed $B_{0}$ and $C_{0}^{(3)}$.

A special situation is when the pull-back of $d C^{(3)}$ to the fivebrane world-volume vanishes, in which case there exists a two-form $C^{(2)}$ on the world-volume such that $C^{(3)}=d C^{(2)}$, and we can take $B=C^{(2)}+B^{\prime}$. The second term in (2.1) then simply reads $\int\left|d B^{\prime}\right|^{2}$. The only cases we will encounter where the pull-back of $d C^{(3)}$ is nonvanishing is when we consider one fivebrane in the background of another five-brane. A five-brane induces a background geometry with a non-trivial $d C^{(3)}$ which is, roughly speaking, the unit volume form on the four-spheres surrounding the five-brane in the five 
dimensions transversal to it [25]. When this happens, the kinetic terms for the scalars $u_{\alpha}$ will also be modified because $C_{m z \bar{z}}^{(3)}$ contains terms $\partial_{m} u_{\alpha} \partial_{\alpha} X^{i} \partial_{z} X^{j} \partial_{\bar{z}} X^{k} C_{i j k}^{(3)}$.

A final observation is one regarding the additional scalar fields coming from $B$. As we mentioned previously, if the Riemann surface $\Sigma$ has a component $\Sigma_{0}$ of finite volume we get an additional scalar $\sigma$ by taking $B_{z \bar{z}}=\sigma\left(x^{m}\right) \omega_{z \bar{z}}$, with $\omega_{z \bar{z}}$ the volume form on $\Sigma_{0}$. It turns out that $\sigma$ is a compact scalar. To see this, we replace $\mathbf{R}^{4}$ by $\mathbf{R}^{3} \times S^{1}$. According to [24], in order to be able to define the five-brane partition function, we do not only have to mod out by the gauge transformations $\delta B=C^{(2)}, \delta C^{(3)}=d C^{(2)}$, but also by 'large' gauge transformations where we add an element of $H^{3}\left(\mathbf{R}^{3} \times S^{1} \times \Sigma, \mathbf{Z}\right)$ to both $d B$ and $C^{(3)}$. These large gauge transformations show that we should identify $\sigma$ with $\sigma+$ const.

\section{$3 \quad N=2$ Coulomb Branch}

In [5], Witten constructed configurations of the $M$ theory fivebrane starting from the configurations of D4, NS5 and D6 branes in Type IIA string theory that describe at long distances the dynamics of $N=2$ supersymmetric gauge theories in four dimensions. The Type IIA configuration is in a flat ten-dimensional space-time with time and space coordinates $x^{0}$ and $x^{1}, \ldots, x^{9}$, and the D4, NS5 and D6 branes span the directions 01236, 012345 and 0123789 respectively.

The $M$ theory configuration for the $S U\left(N_{c}\right)$ gauge theory with $N_{f}$ fundamental hypermultiplets $(N=2 \mathrm{SQCD})$ is a fivebrane embedded in the eleven-dimensional space-time $\mathbf{R}^{7} \times S$ where $S$ is the $A_{N_{f}-1}$-type Taub-NUT space which is a four-dimensional noncompact hyper-Kähler manifold. The $\mathbf{R}^{7}$ spans the 0123789 directions, while $S$ spans the 456 directions in the Type IIA limit and wraps on the circle in the eleventh direction. Choosing one of its complex structures, $S$ is described by a resolution of the complex surface

$$
x y=\Lambda^{2 N_{c}-N_{f}} \prod_{i=1}^{N_{f}}\left(v+m_{i}\right),
$$

and is provided with the nowhere vanishing holomorphic two-form

$$
\Omega=\ell_{11}^{3} \mathrm{~d} v \wedge \frac{\mathrm{d} y}{y} .
$$

The parameters $m_{i}$ and the parameters of the resolution (the size of the resulting twospheres) determine the location of the D6 branes in the 45 and 6 directions respectively, where $m_{i}$ 's correspond to the quark bare mass but the parameters of the resolution have no counterpart in the standard gauge theory. $\Lambda$ is a parameter corresponding to the dynamical scale. 
The fivebrane for a theory at a point in the Coulomb branch is $\mathbf{R}^{4} \times \Sigma$ where $\mathbf{R}^{4}$ spans the first four directions 0123 while $\Sigma$ is at a point in the last three directions 789 of $\mathbf{R}^{7}$ and is embedded as a holomorphic curve in $S$. The embedding is given by

$$
x+y=2 C_{N_{c}}\left(v, u_{\alpha}\right):=2\left(v^{N_{c}}+\sum_{\alpha=2}^{N_{c}} u_{\alpha} v^{N_{c}-\alpha}\right) .
$$

This is the same as the Seiberg-Witten curve [6, 26-29] of $N=2$ SQCD and, therefore, the worldvolume theory has the same effective gauge coupling as $N=2$ SQCD [32]. A BPS state is a supersymmetric membrane ending on the fivebrane worldvolume [3,5] whose mass is given by the membrane tension $\ell_{11}^{-3}$ times the area of the spacial part $D$ of its worldvolume. By the condition of the supersymmetric cycle, the area is the same as the integration of the holomorphic two-form $\Omega$ and the mass is given by

$$
\frac{1}{\ell_{11}^{3}}\left|\int_{D} \Omega\right|=\left|\int_{D} \mathrm{~d} v \wedge \frac{\mathrm{d} y}{y}\right|=\left|\oint_{\partial D} v \frac{\mathrm{d} y}{y}\right|,
$$

which agrees with the BPS mass formula for the $N=2$ SQCD, as shown in $[33,34,30]$ for the $N_{f}=0$ cases.

From the fact that the worldvolume theory has the same effective gauge coupling as $N=2$ SQCD, it follows that they have the same Kähler metric on the Coulomb branch as well because of the $N=2$ supersymmetry in four dimensions which both theories possess. We now explicitly check this. ${ }^{1}$

Since we are considering a variation of the holomorphic curve $\Sigma$ with a single noncompact component, the Kähler metric is simply read off from the second order variation of the term $\ell_{11}^{-6} \int \sqrt{-g} \mathrm{~d}^{6} x$ of the fivebrane lagrangian. Recalling that $\Sigma$ is at a point in the 789 directions and is embedded in the complex surface $S$, we find that it is given by formula (2.11). Namely

$$
K_{\alpha \bar{\beta}}=\frac{1}{\ell_{11}{ }^{6}} \int_{\Sigma} \mathrm{d}^{2} z\left(G_{i \bar{j}} G_{k \bar{l}}-G_{i \bar{l}} G_{k \bar{j}}\right) \partial_{z} X^{i} \partial_{\bar{z}} X^{\bar{j}} \frac{\partial X^{k}}{\partial u_{\alpha}} \frac{\partial X^{\bar{l}}}{\partial \bar{u}_{\bar{\beta}}}
$$

where $G_{i \bar{j}}$ denotes the Kähler metric of $S$. Note that $G_{i \bar{j}} G_{k \bar{l}}-G_{i \bar{l}} G_{k \bar{j}}$ is the $i \bar{j} k \bar{l}$ component of the square of the Kähler form $\omega$ of $S$. In the present case where $S$ is a Ricci-flat Kähler manifold of dimension two, the square of the Kähler form is given by

$$
\omega^{2}=\Omega \wedge \bar{\Omega}
$$

We now fix the coordinates of the space-time $S$ and the worldvolume $\Sigma$. As the coordinates of $S$ we can use $v$ and $y$ which are good in the neighborhood of $\Sigma$ except at a subset of

\footnotetext{
${ }^{1}$ Essentially the same computation was recently done independently in [30,31].
} 
measure zero. As the worldvolume coordinate, we choose $v$. This choice of coordinates corresponds to considering the curve $\Sigma$ as the two-sheeted cover of the $v$-plane given by

$$
y^{2}-2 C_{N_{c}}\left(v, u_{\alpha}\right) y+\Lambda^{2 N_{c}-N_{f}} \prod_{i=1}^{N_{f}}\left(v+m_{i}\right)=0 .
$$

Then, there is only one non-trivial component of $\partial X^{k} / \partial u_{\alpha}$ which is

$$
\frac{\partial y}{\partial u_{\alpha}}=y \frac{v^{N_{c}-\alpha}}{y-C_{N_{c}}(v)} .
$$

Note that

$$
\omega_{\alpha}=\frac{v^{N_{c}-\alpha}}{y-C_{N_{c}}(v)} \mathrm{d} v, \quad \alpha=2, \ldots, N_{c}
$$

form a base of the holomorphic differentials of the curve $\Sigma$ of genus $N_{c}-1$. The Kähler metric is then expressed as

$$
\begin{aligned}
K_{\alpha \bar{\beta}} & =\frac{1}{\ell_{11}{ }^{6}} \int_{\Sigma} \mathrm{d}^{2} v \Omega_{v y} \bar{\Omega}_{\bar{v} \bar{y}} \frac{\partial y}{\partial u_{\alpha}} \frac{\partial \bar{y}}{\partial \bar{u}_{\bar{\beta}}}=\int_{\Sigma} \mathrm{d}^{2} v \frac{v^{N_{c}-\alpha}}{y-C_{N_{c}}(v)} \overline{\left(\frac{v^{N_{c}-\bar{\beta}}}{y-C_{N_{c}}(v)}\right)} \\
& =\int_{\Sigma} \omega_{\alpha} \wedge \bar{\omega}_{\bar{\beta}}
\end{aligned}
$$

This is nothing but the Kähler metric of the special geometry which agrees with the field theory knowledge. In order to see this in the standard notation, let us choose a symplectic basis of the first homology class of $\Sigma ; A_{i}, B^{j}\left(i, j=1, \ldots, N_{c}-1\right)$. Then, the $a$ and $a_{D}$ fields are given by $\partial a_{i} / \partial u_{\alpha}=\oint_{A_{i}} \omega_{\alpha}$ and $\partial a_{D}^{j} / \partial u_{\beta}=\oint_{B^{j}} \omega_{\beta}$. Using the Riemann bilinear identity, (3.10) is expressed as

$$
K_{\alpha \bar{\beta}}=\sum_{i=1}^{N_{c}-1}\left(\frac{\partial a_{i}}{\partial u_{\alpha}} \frac{\partial \overline{a_{D}^{i}}}{\partial \bar{u}_{\bar{\beta}}}-\frac{\partial \bar{a}_{i}}{\partial \bar{u}_{\bar{\beta}}} \frac{\partial a_{D}^{i}}{\partial u_{\alpha}}\right) .
$$

In this way, we have obtained the standard form of the scalar kinetic term of the effective lagrangian

$$
S_{\text {kin }}=\operatorname{Im} \int \mathrm{d}^{4} x \eta^{m n} \sum_{i=1}^{N_{c}-1} \partial_{m} a_{i} \partial_{n} \overline{a_{D}^{i}}
$$

Note that the essential point for obtaining the special geometry is the holomorphic anti-holomorphic factorization (3.6) of the square of the Kähler form. This would not be the case if $\Sigma$ were embedded in a Calabi-Yau three-fold (as in the case of $N=1$ SQCD) nor in a Ricci-non-flat complex surface in the space-time (as in the case we will consider in section 5). 


\section{$4 \quad N=2$ Higher Derivative Terms}

In this section we will consider the four-derivative terms for the scalar fields in $N=2$ SYM theory, both from the field theory point of view and from the brane point of view, and compare the results.

\subsection{Field Theory}

The low-energy effective action of $N=2$ SYM theory can in $N=2$ superspace be written as

$$
S=\int d^{4} x d^{4} \theta F\left(A^{i}\right)+\int d^{4} x d^{4} \bar{\theta} \bar{F}\left(\bar{A}^{i}\right)+\int d^{4} x d^{4} \theta d^{4} \bar{\theta} H\left(A^{i}, \bar{A}^{\bar{i}}\right)+\ldots,
$$

where $F$ is a holomorphic function and $A^{i}\left(\bar{A}^{\bar{i}}\right)$ are abelian $N=2$ chiral (antichiral) vector superfields. The real function $H\left(A^{i}, \bar{A}^{\bar{i}}\right)$ is the one that gives rise to four derivative terms for the scalars in the low-energy effective action. We denote by $\Phi^{i}$ and $W_{\alpha}^{i}$ the $N=1$ chiral superfield and $N=1$ field strength that are contained in $A^{i}$, and by $\phi^{i}$ the complex scalar in $\Phi^{i}$. The kinetic term for the $\phi^{i}$ is

$$
S=\int d^{4} x\left(\partial_{m} \phi^{i} \partial^{m} \phi^{\bar{j}}\right) K_{i \bar{j}}
$$

where $K_{i \bar{j}} \sim \operatorname{Im}\left(F_{i j}\left(\phi^{i}\right)\right)$ (subscripts on $F$ and $H$ denote derivatives with respect to $\phi^{i}$ ).

The function $H$ been studied in [36-43]. Some general facts such as its behavior under $S L(2, \mathbf{Z})$ were discussed in [36], and its asymptotic behavior was discussed in [40,41]. Several contributions to $H$ are known explicitly, such as the one-loop contribution [39], the two-loop contribution [43] (which vanishes), the one-instanton contribution [38] and the two-instanton contribution [42]. An exact form for $H$ in the case of $S U(2)$ was conjectured in [37]. In terms of the gauge invariant coordinate $u$ on the Coulomb branch of the $S U(2)$ gauge theory it states that

$$
H(u, \bar{u})=c\left|u^{2}-\Lambda_{N=2}^{4}\right|\left(K_{u \bar{u}}\right)^{2},
$$

where $c$ is some constant. $H(u, \bar{u})$ should really be seen as a function of $A, \bar{A}$ rather than $u, \bar{u}$, because a holomorphic function of an $N=2$ vector superfield is in general no longer an $N=2$ vector superfield. Such a function is still chiral, but no longer satisfies the Bianchi identity. The one-loop results for $S U(2)$ are

$$
K_{u \bar{u}} \sim \frac{\log \left(16 u \bar{u} / \Lambda_{N=2}^{4}\right)}{\sqrt{u \bar{u}}}, \quad u=A^{2} / 2, \quad H(A, \bar{A}) \sim \log \left(A / \Lambda_{N=2}\right) \log \left(\bar{A} / \Lambda_{N=2}\right) .
$$


In addition, there are instanton correction that are typically weighted with factors $\left(\Lambda_{N=2}^{4} / u^{2}\right)$, but we will not consider those here.

As the brane will not give us an answer in superspace but in components, in order to be able to compare we need to work out what (4.1) looks like in components. Using the $N=1$ expansion of (4.1) in e.g. [36] or [40], we find the following four-derivative terms for the scalars $\phi^{i}$

$$
\begin{aligned}
S_{4}=\int & d^{4} x\left(2 H_{i \bar{j}}\left(\partial^{m} \partial_{m} \phi^{i}\right)\left(\partial^{n} \partial_{n} \phi^{\bar{j}}\right)+H_{i j \bar{k}}\left(\partial^{m} \phi^{i}\right)\left(\partial_{m} \phi^{j}\right)\left(\partial^{n} \partial_{n} \phi^{\bar{k}}\right)\right. \\
& \left.+H_{\bar{i} \bar{j} k}\left(\partial^{m} \phi^{\bar{i}}\right)\left(\partial_{m} \phi^{\bar{j}}\right)\left(\partial^{n} \partial_{n} \phi^{k}\right)+H_{i j \bar{k} \bar{l}}\left(\partial^{m} \phi^{i}\right)\left(\partial_{m} \phi^{j}\right)\left(\partial^{n} \phi^{\bar{k}}\right)\left(\partial_{n} \phi^{\bar{l}}\right)\right) .
\end{aligned}
$$

In deriving this result we used several partial integrations, and put the auxiliary fields equal to zero. If the fermions are equal to zero that is allowed because there are no terms linear in the auxiliary fields that couple only to the scalar fields $\phi^{i}$. This can easily be understood from the fact that the scalars $\phi^{i}$ are invariant under the global $S U(2)_{R}$ transformations, whereas the three auxiliary fields form a triplet. We can simplify the structure of the four-derivative term even further by making some field redefinitions of the $\phi^{i}$. The field equation for $\phi^{i}$ reads

$$
\partial^{m} \partial_{m} \phi^{i}=-\left(K_{i \bar{j}}\right)^{-1} K_{\bar{j} k l}\left(\partial^{m} \phi^{k}\right)\left(\partial_{m} \phi^{l}\right)
$$

and using field redefinitions we can replace $\partial^{m} \partial_{m} \phi^{i}$ in (4.5) by the right hand side of (4.6). This then finally leads to the following expression for the four-derivative term

$$
S_{4}=\int d^{4} x\left(\tilde{H}_{i j \bar{k} \bar{l}}\left(\partial^{m} \phi^{i}\right)\left(\partial_{m} \phi^{j}\right)\left(\partial^{n} \phi^{\bar{k}}\right)\left(\partial_{n} \phi^{\bar{l}}\right)\right)
$$

where

$$
\tilde{H}_{i j \bar{k} \bar{l}}=H_{i j \bar{k} \bar{l}}-H_{i j \bar{p}}\left(K_{\bar{p} q}\right)^{-1} K_{q \bar{k} \bar{l}}-K_{i j \bar{p}}\left(K_{\bar{p} q}\right)^{-1} H_{q \bar{k} \bar{l}}+2 K_{i j \bar{p}}\left(K_{\bar{p} q}\right)^{-1} H_{q \bar{r}}\left(K_{\bar{r} s}\right)^{-1} K_{s \bar{k} \bar{l}} .
$$

It is quite interesting that the four-derivative terms can be brought in the simple form (4.7), a fact that the brane knows about as we will see in the next section. For $S U(2)$, we find that the semi-classical four-derivative term reads

$$
S=\int d^{4} x\left(\partial^{m} u \partial_{m} u\right)\left(\partial^{n} \bar{u} \partial_{n} \bar{u}\right) \frac{8+4 \log y+(\log y)^{2}}{u^{2} \bar{u}^{2}(\log y)^{2}}, \quad y=\frac{16 u \bar{u}}{\Lambda_{N=2}^{4}} .
$$

\subsection{Fivebrane}

Let us now compute the higher derivative terms using the fivebrane ${ }^{1}$. They can be extracted by expanding (2.5) in powers of $L$. We will concentrate on the case of a pure

\footnotetext{
${ }^{1}$ These terms were also recently computed in [31]
} 
gauge theory without matter, with target space metric as given in (A.1). Recall that $\Sigma$ is described by

$$
t^{2}-2 t C\left(v, u_{\alpha}\right)+\Lambda_{N=2}^{2 N_{c}}=0, \quad C\left(v, u_{\alpha}\right)=v^{N_{c}}+\sum_{\alpha=2}^{N_{c}} u_{\alpha} v^{N_{c}-\alpha} .
$$

We identify the complex coordinate $z$ on $\Sigma$ with $v$ and find (where $C \equiv C\left(v, u_{\alpha}\right)$ and $\left.C^{\prime} \equiv \partial C\left(v, u_{\alpha}\right) / \partial v\right)$

$$
\begin{aligned}
g_{z \bar{z}} & =\frac{\ell_{s t}^{4}|t-C|^{2}+R^{2}\left|C^{\prime}\right|^{2}}{|t-C|^{2}} \\
g_{\alpha \bar{\beta}} & =\frac{R^{2} v^{N_{c}-\alpha} \bar{v}^{N_{c}-\bar{\beta}}}{|t-C|^{2}} \\
g_{z \bar{\beta}} & =\frac{R^{2} C^{\prime} \bar{z}^{N_{c}-\bar{\beta}}}{|t-C|^{2}} \\
g_{\alpha \bar{z}} & =\frac{R^{2} \bar{C}^{\prime} z^{N_{c}-\alpha}}{|t-C|^{2}} .
\end{aligned}
$$

From this we obtain

$$
L_{m n}=\left(\partial_{m} u^{\alpha} \partial_{n} \bar{u}^{\bar{\beta}}\right) \frac{\ell_{11}^{6} v^{N_{c}-\alpha} \bar{v}^{N_{c}-\bar{\beta}}}{\ell_{s t}^{4}|t-C|^{2}+R^{2}\left|C^{\prime}\right|^{2}}+(m \leftrightarrow n) .
$$

The five-brane action (2.5) can now be rewritten in the following convenient form: introduce $f, d$ defined by

$$
\begin{aligned}
& f=\left(\partial^{m} u^{\alpha} \partial_{m} u^{\beta}\right) \frac{\ell_{11}^{6} v^{2 N_{c}-\alpha-\beta}}{\ell_{s t}^{4}|t-C|^{2}+R^{2}\left|C^{\prime}\right|^{2}} \\
& d=\left(\partial^{m} u^{\alpha} \partial_{m} \bar{u}^{\bar{\beta}}\right) \frac{\ell_{11}^{6} v^{N_{c}-\alpha} \bar{v}^{N_{c}-\bar{\beta}}}{\ell_{s t}^{4}|t-C|^{2}+R^{2}\left|C^{\prime}\right|^{2}}
\end{aligned}
$$

then

$$
S=\frac{1}{\ell_{11}^{6}} \int d^{4} x d^{2} v \frac{\ell_{s t}^{4}|t-C|^{2}+R^{2}\left|C^{\prime}\right|^{2}}{|t-C|^{2}} \sqrt{(1+d)^{2}-f \bar{f}} .
$$

This expression makes it clear what kind of higher derivative terms can appear in the fivebrane action, and what their relative coefficients are. In particular, the four-derivative term comes only from the $f \bar{f}$ term in (4.14), and it is therefore precisely of the same form as (4.7), namely

$$
S_{4}=\int d^{4} x \tilde{H}_{\alpha \beta \bar{\gamma} \bar{\delta}}\left(\partial^{m} u_{\alpha} \partial_{m} u_{\beta}\right)\left(\partial^{n} \bar{u}_{\bar{\gamma}} \partial_{n} \bar{u}_{\bar{\delta}}\right)
$$

with

$$
\tilde{H}_{\alpha \beta \bar{\gamma} \bar{\delta}}=-\frac{1}{2} \int_{\Sigma} d^{2} v \frac{\ell_{11}^{6} v^{2 N_{c}-\alpha-\beta} \bar{v}^{2 N_{c}-\bar{\gamma}-\bar{\delta}}}{|t-C|^{2}\left(\ell_{s t}^{4}|t-C|^{2}+R^{2}\left|C^{\prime}\right|^{2}\right)}
$$


We see that the fivebrane provides a form of the four-derivative term that is consistent with the general form of a four-derivative term in an $N=2$ field theory, provided we make certain field redefinitions. In itself this is not surprising, as the fivebrane theory does have $N=2$ supersymmetry in four dimensions, and should therefore be consistent with the most general supersymmetric field theory. The fact that we have to perform certain field redefinitions is presumably related to the fact that the $N=2$ supersymmetry in field theory is realized differently than it is in the fivebrane theory. The latter is known to be realized in a highly non-linear way [44]. The fact that in the fivebrane theory fewer higher derivative terms appear than in the field theory, suggests that the non-linear realization of supersymmetry on the fivebrane is a very efficient way to organize the higher derivative terms, and perhaps also the fermionic terms, in $N=2$ effective actions.

\subsection{Comparison}

The first thing that one notices in the brane result (4.16) is the fact that it depends non-trivially on $R$ and $\ell_{11}$, and that it therefore cannot be equivalent to the field theory answer. This is the first time in this paper that we compare a field theory quantity that is not protected by holomorphy or global symmetries to the same quantity obtained from the brane. The brane result depends explicitly on $R$ and $\ell_{11}$ and there is no obvious limit for these quantities that yields a sensible result. The four-derivative term is ill-defined both in the limit that $R$ goes to zero and that $R$ goes to infinity. Therefore, the brane and field theory results agree only for processes involving very low energies and momenta. Certain qualitative features of the higher derivative terms do agree. Consider for instance the points in moduli space where (4.16) becomes singular. This can only happen when the denominator in the integrand behaves like $\left|v-v_{0}\right|^{k}$ near some point $v=v_{0}$ and $k \leq 2$. There are two possibilities, either $(t-C)^{2}$ has a double zero or $(t-C)^{2}$ and $C^{\prime}$ have a common zero. The second condition implies the first one, and $(t-C)^{2}$ has a double zero only at the singularities in the moduli space where a dyon becomes massless. This is consistent with what one expects from field theory. It is in this sense that one might consider the theories to be in the same universality class. To get an idea to what extent the results are quantitatively different, we compare the semiclassical field theory result (4.9) with the brane result for $S U(2)$, which reads (see (4.16))

$$
\tilde{H}_{u u \bar{u} \bar{u}} \sim \int d^{2} v \frac{\ell_{11}^{6}}{\left|\left(v^{2}+u\right)^{2}-\Lambda_{N=2}^{4}\right|\left(\ell_{s t}^{4}\left|\left(v^{2}+u\right)^{2}-\Lambda_{N=2}^{4}\right|+4 R^{2}|v|^{2}\right)} .
$$


The semiclassical region corresponds to large $u$. If $|u| \gg R^{2} \ell_{s t}^{-4}$ and $|u| \gg \Lambda_{N=2}^{2}$, then one can easily show that

$$
\left|\tilde{H}_{u u \bar{u} \bar{u}}\right| \geq \frac{1}{4} \int_{|v| \geq \ell_{s t}^{2} R^{-1}|u|} d^{2} v \frac{R^{2}}{|v|^{8}} \sim R^{8} \ell_{s t}^{-12}|u|^{-6} .
$$

For these values of $|u|$ the integral over the disc $|v| \leq R \ell_{s t}^{-2}$ contributes something of the order $R^{4} \ell_{s t}^{-4}|u|^{-4}$ to $\left|\tilde{H}_{u u \bar{u} \bar{u}}\right|$. Altogether it is not clear whether (4.16) does have the right $|u|^{-4}$ behavior, but if it does, the coefficient in front of $|u|^{-4}$ will almost certainly depend on $R / \ell_{s t}$. Thus, even in the semiclassical regime, the field and brane theories are quantitatively quite different.

\section{$4.4 S U\left(N_{c}\right)$ with $N_{f}>0$}

In the presence of matter fields, field theory makes a few interesting non-trivial predictions. The one-loop contribution to $H\left(A^{i}, \bar{A}^{i}\right)$ has a coefficient proportional to $2 N_{f}-N_{c}$. In addition, there are indications $[35,43]$ that $H\left(A^{i}, \bar{A}^{i}\right)$ receives no perturbative corrections beyond one-loop at all. Therefore, in the case where $N_{c}=2 N_{f}$, the function $H\left(A^{i}, \bar{A}^{i}\right)$ should receive contributions only from instantons.

Another prediction form field theory is that in the finite, scale invariant case $N_{f}=2 N_{c}$, the one-loop result for $H\left(A^{i}, \bar{A}^{i}\right)$ is exact [45].

To see whether we can reproduce these results from the fivebrane, we have to work out the four-derivative term in the presence of matter. Matter is included in the $M$ theory framework by modifying space-time to include a multi-Taub-NUT space. The Taub-NUT space depends on several parameters $\left(x_{i}^{4}, x_{i}^{5}, x_{i}^{6}\right)$, corresponding to the locations of the D6 branes in the Type IIA picture (see Appendix B). Two of the three parameters, $x_{i}^{4}$ and $x_{i}^{5}$, correspond to the mass of a quark and should be taken equal to zero, but the other parameters $x_{i}^{6}$ are free. They do not correspond to parameters in field theory. If we work out the four-derivative term using the Taub-NUT background we find that the result does depend on $x_{i}^{6}$. This illustrates once more the difference between the brane results and field theory. A limit that is particularly easy to analyze is to send all $x_{i}^{6} \rightarrow \infty$. From the Type IIA point of view, this corresponds to using semi-infinite four-branes to realize the matter. The result one obtains is given by (4.15) and (4.16), where in (4.16) $\left|C^{\prime}\right|^{2}$ should be replaced by $\left|\frac{t-C}{t} \frac{\partial t}{\partial v}\right|^{2}$. This result deviates considerably from the field theory predictions. For $N_{c}=2 N_{f}$ there are still one-loop contributions to $H\left(A^{i}, \bar{A}^{i}\right)$, and for $N_{f}=2 N_{c}$ the one-loop result is not exact. In the latter case, the field theory result that the one-loop result is exact was obtained in [45] using global symmetries and a scaling argument. The reason that the same argument does not apply to the brane calculation 
is that the fivebrane depends on two additional parameters, $R$ and $\ell_{s t}$. To have the same scale invariance as in [45] we should assign weight -1 to both $R$ and $\ell_{s t}$, and there are many new scale invariant four-derivative terms that one can write down that do depend on $R$ and $\ell_{s t}$.

\section{$5 \quad N=1$ Coulomb Branch}

In this section, we consider an example of a worldvolume theory with $N=1$ supersymmetry in four-dimensions where we can obtain the Kähler metric of the moduli space exactly within the eleven-dimensional supergravity approximation of $M$ theory .

The example we consider corresponds to the $N=1$ supersymmetric gauge theory with gauge group $S U\left(N_{c}\right)$ with a massless adjoint chiral multiplet $\Phi$ and $N_{f}$ quark multiplets $Q^{i}, \widetilde{Q}_{i}$ (fundamental and anti-fundamental chiral multiplets) with bare mass $m$. The classical Lagrangian of the theory is the standard D-term plus the F-term given by the superpotential

$$
W=m \sum_{i=1}^{N_{f}} \widetilde{Q}_{i} Q^{i} .
$$

We do not turn on the Yukawa coupling $\widetilde{Q} \Phi Q$ which makes the system $N=2$ supersymmetric. The classical moduli space of vacua consists of a single Coulomb branch where the quark VEVs are zero $Q=\widetilde{Q}=0$ and $\Phi$ is diagonal. At energies below $m$ the field content of the theory is the same as that of $N=2$ super-Yang-Mills theory with gauge group $S U\left(N_{c}\right)$, but the quark multiplets introduce $N=2$ breaking interactions and we do not expect to obtain the special geometry as the quantum moduli space of vacua. If the bare mass $m$ is very large, however, the $N=2$ breaking interactions are suppressed as inverse powers of $m$, and we do expect the moduli space to be a small deformation of the special geometry for the $N=2$ super-Yang-Mills theory with gauge group $S U\left(N_{c}\right)$. We compute the corresponding deformation in the worldvolume theory, and compare with what we expect from field theory.

There is no a priori reason to expect that these two agree because the supergravity computation is valid when all the characteristic lengths of the space-time and the brane are much larger than the Planck length while the worldvolume theory becomes close to the four-dimensional gauge theory only in the Type IIA limit where the radius of the circle in the eleventh direction is much smaller than $\ell_{11}$. As in the $N=2$ Coulomb branch, the effective gauge coupling constant will be correctly reproduced because abelian gauge fields are obtained by the chiral two form on the fivebrane whose lagrangian is scale invariant and the result will persist to be true even if we scale the system down to the Type IIA 
region $R \ll \ell_{11}$. However, because of the lack of $N=2$ supersymmetry it does not mean in the present case that the Kähler metric is also correctly reproduced.

As we will see, there is indeed a clear discrepancy between the two. The reason we dare to carry out this computation is that the result might be useful for the following two purposes. In the region $R \ll \ell_{11}$ the discrepancy would mainly be due to the correction to the supergravity approximation of $M$ theory which is still in rather mysterious, and it can be used to learn about $M$ theory itself. In the region $R \gg \ell_{11}$, the discrepancy originates in the effects of the degrees of freedom which are not part of the four-dimensional gauge theory. If we want to learn something about field theory from the worldvolume theory of the brane in general, it would be very useful to estimate the effects of such extra degrees of freedom as accurately as possible. The present computation may be considered as the first step towards obtaining some quantitative information about them.

\subsection{Brane Construction}

We start by constructing a Type IIA brane configuration whose worldvolume dynamics describes at long distances the $N=1$ gauge theory given above. It involves $N_{c} \mathrm{D} 4$ branes stretched between two NS 5-branes with $N_{f}$ D6 branes located away from them. The worldvolume of D4, NS5 and D6 branes span the directions 01236, 012389 and 0123789 respectively, where the two NS 5-branes are at a point $v=0, x^{7}=0$ in the 457 directions and are separated in the $x^{6}$ direction, the D4 branes stretched between them are at points in the 89 directions, and the D6 branes are at $v=-m, x^{6}=0$. Note that the $\mathrm{D} 4$ and NS5 branes are separated from the D6 branes in the 45 directions by $\Delta v=m$. This configuration is obtained from that of $N=2 \mathrm{SQCD}$ with $N_{f}$ massive quarks by a 90 degree rotation of the D4/NS5 system in the 45-89 directions while keeping the D6 branes intact. The configuration is invariant under the groups $U(1)_{45}$ and $U(1)_{89}$ of rotations in

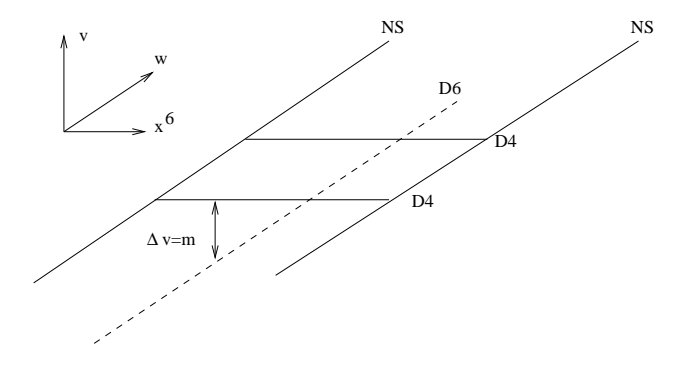

Figure 1: The Type IIA Configuration

the 45 and 89 directions provided we assign a suitable $U(1)_{45}$ charge to $m$. The coordinates 
$v$ and $x^{8}+i x^{9}$ carry charge $(2,0)$ and $(0,2)$ respectively under $U(1)_{45} \times U(1)_{89}$, and $m$ must be assigned a charge $(2,0)$.

The open strings stretched between the D4 branes create a $U\left(N_{c}\right)$ vector multiplet in five dimensions with sixteen supersymmetry, but the boundary condition at the ends on the NS 5-branes projects this to an $N=2 S U\left(N_{c}\right)$ vector multiplet in four dimensions which contains in $N=1$ language the vector multiplet $W_{\alpha}$ and the adjoint chiral multiplet $\Phi$. The rotation symmetry $U(1)_{45} \times U(1)_{89}$ is identified with the R-symmetry under which $\Phi$ carries charge $(0,2)$ and gluino carries charge $(1,1)$. Open strings stretched between D4 and D6 branes create $N_{f}$ fundamental and anti-fundamental chiral multiplets which carry $U(1)_{45} \times U(1)_{89}$ R-charge $(0,1)$. As in the three-dimensional analog [57], one can show that the superpotential invariant under the R-symmetry is $W=m \widetilde{Q} Q$.

It is easy to lift this to an $M$ theory configuration. It is a configuration of a single fivebrane in the space-time $\mathbf{R}^{7} \times S$ where $S$ is a Taub-NUT space which has a complex structure (among others) described by the equation $x y=\Lambda_{N=1}^{2 N_{c}-N_{f}}(v+m)^{N_{f}}$. We have introduced in advance a parameter $\Lambda_{N=1}$ that characterize the distance between the two NS 5-brane. The fivebrane is of the form $\mathbf{R}^{4} \times \Sigma$ where $\Sigma$ is located at $x^{7}=0$ and is embedded as a holomorphic curve in the space $\left.S\right|_{v=0} \times \mathbf{R}^{2}$. Here, $\left.S\right|_{v=0}$ is the $v=0$ locus of $S$ which is the cylinder described by

$$
x y=m^{N_{f}} \Lambda_{N=1}^{2 N_{c}-N_{f}},
$$

while $\mathbf{R}^{2}$ is the 89 directions of the space-time in which we introduce a complex coordinate ${ }^{1}$

$$
W=\ell_{s t}{ }^{-2}\left(x^{8}+i x^{9}\right)
$$

The curve has two regions with large $w$ corresponding to the two NS 5-branes, and these obey the boundary condition $x \sim W^{N_{c}}$ in one region of large $w$ and $y \sim{ }^{N_{c}}$ in the other. The embedding satisfying this condition is given by

$$
x+y=2 C_{N_{c}}\left(w, u_{\alpha}\right)=2\left(W^{N_{c}}+\sum_{\alpha=2}^{N_{c}} u_{\alpha} W^{N_{c}-\alpha}\right),
$$

where $u_{\alpha}$ 's are parameters characterizing the distance between the $N_{c}$ D4-branes.

The configuration is invariant under $U(1)_{45} \times U(1)_{89}$ if we assign charge $\left(0,2 N_{c}\right)$ to $x$ and $y,\left(-2 N_{f}, 4 N_{c}\right)$ to $\Lambda_{N=1}^{2 N_{c}-N_{f}}$, and $(0,2 \alpha)$ to $u_{\alpha}$. From this we can identify $\Lambda_{N=1}$ and

\footnotetext{
${ }^{1}$ This should not be confused with the coordinate $w=\ell_{11}{ }^{-3}\left(x^{8}+i x^{9}\right)$ which is used in other parts of this paper. The difference in the prefactor is because of the difference in the identification of the parameters of branes and fields.
} 
the $u_{\alpha}$ 's as the dynamical scale of the gauge theory and the color Casimirs $\operatorname{det}(w+\Phi)=$ $C_{N_{c}}\left(w, u_{\alpha}\right)$ at some cut-off scale. The effective gauge coupling constant of the abelian gauge theory on the brane is given by the period matrix of the curve. The curve we have obtained reproduces the effective coupling of the gauge theory of interest given by [46].

For large values of $m$, the theory at energies below $m$ is approximately the $N=2$ pure Yang-Mills theory. The dynamical scale of the low energy theory $\Lambda_{N=2}$ is related to the high energy theory by

$$
\Lambda_{N=2}^{2 N_{c}}=\left(\frac{g_{L}^{2}}{g_{H}^{2}}\right)^{N_{c}} m^{N_{f}} \Lambda_{N=1}^{2 N_{c}-N_{f}},
$$

where $g_{L}$ and $g_{H}$ are the gauge coupling constants of the low and high energy theories at the cut-off scale. The factor $\left(g_{L}^{2} / g_{H}^{2}\right)^{N_{c}}$ appears [47] since the kinetic term for $\Phi$ is given by

$$
\frac{1}{g^{2}} \int \mathrm{d}^{4} \theta \operatorname{Tr}\left(\Phi^{\dagger} \Phi\right),
$$

because the W-boson mass is given by the separation of the eigenvalues of $\Phi$, not multiplied by $g$. Likewise, the field $\Phi$ gets renormalized as we flow down the energy below $m$, and the low energy field $\Phi^{(2)}$ is related to the high energy field by $\Phi^{(2)}=\left(g_{L} / g_{H}\right) \Phi$. In terms of these variables, the curve is given by

$$
\tilde{x}+\tilde{y}=2 C_{N_{c}}\left(\tilde{W}, u_{\alpha}^{(2)}\right), \quad \tilde{x} \tilde{y}=\Lambda_{N=2}^{2 N_{c}},
$$

where $u_{\alpha}^{(2)}=\left(g_{L} / g_{H}\right)^{\alpha} u_{\alpha}$ and $\tilde{x}, \tilde{y}, \tilde{w}$ are suitably rescaled coordinates. This is nothing but the curve of $N=2$ super-YM theory, and hence we have shown that the effective gauge coupling constant is the same as the one in the $N=2$ super-Yang-Mills theory. However, due to the absence of $N=2$ supersymmetry, the scalar kinetic term (and hence the Kähler metric on the moduli space) is different as we now see.

\subsection{Kähler Metric on the Moduli Space}

As in the case of the $N=2$ Coulomb branch, the Kähler metric on the moduli space is simply read off from the second order variation of the term $\ell_{11}{ }^{-6} \int \sqrt{-g} \mathrm{~d}^{6} x$ of the fivebrane Lagrangian. It is given by the formula (2.11) or (3.5) where $G_{i \bar{j}} G_{k \bar{l}}-G_{i \bar{l}} G_{k \bar{j}}$ is the $i \bar{j} k \bar{l}$ component of the square of the Kähler form of $\left.S\right|_{v=0} \times \mathbf{R}^{2}$. By using the expression of the Taub-NUT metric given in Appendix B, Equation (B.5), the Kähler form of $\left.S\right|_{v=0} \times \mathbf{R}^{2}$ is given by

$$
\omega=R^{2} U^{-1} \frac{\mathrm{d} y}{y} \wedge \frac{\mathrm{d} \bar{y}}{\bar{y}}+\ell_{s t}{ }^{4} \mathrm{~d} w \wedge \mathrm{d} \bar{w}
$$


where

$$
U=1+\frac{N_{f} R}{2 \sqrt{\left|\ell_{s t}{ }^{2} m\right|^{2}+\left(x^{6}\right)^{2}}} .
$$

The square of $\omega$ is thus

$$
\omega^{2}=\ell_{11}{ }^{6} U^{-1}\left|\mathrm{~d} w \wedge \frac{\mathrm{d} y}{y}\right|^{2} .
$$

Proceeding as in the computation of $N=2$ Coulomb branch, we find that the Kähler metric is given by

$$
K_{\alpha \bar{\beta}}=\int_{\Sigma} U^{-1} \omega_{\alpha} \wedge \bar{\omega}_{\bar{\beta}}
$$

where $\omega_{\alpha}$ are the holomorphic differentials $\omega_{\alpha}={ }^{N_{c}-\alpha} \mathrm{d} w /\left(y-C_{N_{c}}(w)\right)$. This is indeed different from the one of special geometry. However, the prefactor $U^{-1}$ in the integrand is nowhere vanishing nor divergent and is bounded from below and above as $\left(1+N_{f} R M_{s t}^{2} / 2|m|\right)^{-1} \leq U^{-1} \leq 1$, and hence the moduli space has the same type of singularity as in the $N=2$ theory. In particular, the same type of massless particles appear as in the $N=2$ theory. This is what is expected in field theory. Therefore, the brane captures the correct qualitative feature of the field theory of interest.

Let us take a closer look at this metric. The coordinates $y$ and $x$ are related to $x^{6}$ and $x^{10}$ by the formulae (B.4) and (B.7). As in section 4.4, the metric depends on the overall constants in these formulae which is a parameter with no counterpart in field theory. This already shows a discrepancy between the brane and field theory results. We will only examine the metric for one particular value that puts the D6 branes exactly in the middle between the two NS 5-branes in the $x^{6}$ direction. Since all the D6 branes are at $x^{6}=0$, the requirement is $|x|=|y|$ at $x^{6}=0$. This yields at $v=0$ that $y=m^{N_{f} / 2} \Lambda_{N=1}^{N_{c}-N_{f} / 2} t$, $x=m^{N_{f} / 2} \Lambda_{N=1}^{N_{c}-N_{f} / 2} t^{-1}$ where $|t|=1$ at $x^{6}=0$. By introducing the rescaled variable $\widetilde{x}=x^{6} /\left|\ell_{s t}{ }^{2} m\right|, t$ is given by

$$
t=\mathrm{e}^{-\left(\widetilde{m} \widetilde{x}+i x^{10}\right)}\left(\sqrt{1+\widetilde{x}^{2}}-\widetilde{x}\right)^{N_{f} / 2},
$$

where

$$
\widetilde{m}=\frac{|m|}{R M_{s t}^{2}} .
$$

In terms of the new variables, the curve $\Sigma$ is given by

$$
t+t^{-1}=2 C_{N_{c}}\left(w, u_{\alpha}\right) /\left(m^{N_{f} / 2} \Lambda_{N=1}^{N_{c}-N_{f} / 2}\right)
$$

and the function $U$ is expressed as

$$
U=1+\frac{N_{f}}{2 \widetilde{m}}\left(1+\widetilde{x}^{2}\right)^{-\frac{1}{2}} .
$$


Then, we see that the Kähler metric (5.11) depends only on $m^{N_{f}} \Lambda_{N=1}^{2 N_{c}-N_{f}}, u_{\alpha}$, and the combination $\widetilde{m}(5.13)$.

In the limit $m \rightarrow \infty$, the Kähler metric converges to that of the $N=2$ Coulomb branch as is also the case for the gauge theory of interest. However, the precise value of $m$ above which the metric becomes close to the one of the $N=2$ theory is different from the one in the gauge theory. In the gauge theory, provided $m \gg \Lambda_{N=2}$, the metric is close to the $N=2$ metric in the region in which $\langle\Phi\rangle \ll m$. In the worldvolume theory of the brane, however, the metric is close to the $N=2$ metric if $\widetilde{m} \gg 1$, namely,

$$
m \gg R M_{s t}^{2}
$$

irrespective of the values of $u_{\alpha}$.

The structure of deviation from the $N=2$ metric looks also different. In general it is not easy to carry out the integral (5.11). However, for large enough $m$ (5.16), in the region of the moduli space where

$$
\Lambda_{N=2} \ll\langle\Phi\rangle \ll \Lambda_{N=2} \exp \left(\frac{|m|}{N_{c} R M_{s t}^{2}}\right),
$$

$\omega_{\alpha} \wedge \bar{\omega}_{\bar{\beta}}$ is large only for $\widetilde{x} \ll 1$. Then, we can approximate $U$ by its value at $\widetilde{x}=0$ and the Kähler metric (5.11) differs from the $N=2$ metric simply by a constant multiplication:

$$
K_{\alpha \bar{\beta}}=\left(1+\frac{N_{f} R M_{s t}^{2}}{2|m|}\right)^{-1} \cdot \frac{g_{H}^{2}}{g_{L}^{2}} \cdot K_{\alpha \bar{\beta}}^{N=2} .
$$

On the gauge theory side, the deviation is suppressed as powers of $\langle\Phi\rangle / m$ in the region where $\Lambda_{N=2} \ll\langle\Phi\rangle \ll m$. In particular, it is not so simple as (5.18).

Notice that both in section 4 and here the scale $R M_{s t}^{2}$ appears. In particular, it is a natural unit in the brane construction to measure the mass of the quark in, see (5.13). We briefly indicate the origin of this scale. In the fivebrane of $M$ theory, part of the fivebrane corresponds to the D4 brane of Type IIA, and part corresponds to the NS5 brane of Type IIA. The transition region between the two parts is characterized by $\partial\left(\ell_{s t}^{2} v\right) / \partial x^{6} \sim 1$. There are two such transition regions, separated by a distance $\Delta x^{6}$, which for pure gauge theory can be estimated to be $\Delta x^{6}=-2 N_{c} R \log \left(\Lambda_{N=2} / N_{c} R M_{s t}^{2}\right)$. This distance $\Delta x^{6}$ is in the Type IIA picture to be identified with $L_{\text {brane }}$, the distance between the two NS branes. The coupling in the Type IIA picture was given by $1 / g^{2}=L_{\text {brane }} / R$, and using the above results for $\Delta x^{6}$ we see that $\exp \left(-1 / g^{2}\right)=\left(\Lambda_{N=2} / N_{c} R M_{s t}{ }^{2}\right)^{2 N_{c}}$. Thus, $N_{c} R M_{s t}^{2}$ is the scale at which the bare coupling $1 / g^{2}$ is defined. 


\section{$6 \quad N=2$ Higgs Branch}

In sections 4 and 5, we saw that the fivebrane theory on $\mathbf{R}^{4} \times \Sigma$ and the fourdimensional gauge theory give quantitatively different results for the Kähler metric of $N=1$ theories and higher derivative terms of $N=2$ theory. The possible sources of such differences are the Kaluza-Klein modes on the non-compact surface $\Sigma$, which do not have counterparts in the four-dimensional gauge theory, and the issue of the decoupling of the bulk physics. The effect of such extra modes becomes small in the region of the parameter space where there is a large correction to the eleven-dimensional supergravity approximation. However, the computation in the eleven-dimensional supergravity approximation gave a correct answer for the $N=2$ Coulomb branch metric and also for the effective gauge coupling of the $N=2$ and $N=1$ theories in the Coulomb phase. In all these cases, directly or indirectly via supersymmetry, the computation involved the chiral two form of the fivebrane theory which has the Lagrangian given by the second term of (2.1). This suggests that this part of the fivebrane theory is remarkably rigid as we move around in parameter space. In this section, we perform a further test of this observation by studying the Higgs branches of the $N=2$ theory. The metric on these Higgs branches is also related to the chiral two form, and indeed in several cases we can recover the exact field theory metric using a fivebrane calculation.

\subsection{Field Theory}

We will consider four dimensional $N=2$ supersymmetric gauge theory with gauge group $S U\left(N_{c}\right)$ and $N_{f}$ hypermultiplets in the fundamental representation (quarks). The Higgs branch (baryonic branch) of the theory has complex dimension $2 N_{c} N_{f}-2\left(N_{c}^{2}-1\right)$. There are also mixed branches (non baryonic branches) labeled by an integer $r$ [48] with complex dimension $2 r\left(N_{f}-r\right)$. The metrics on the Higgs branches of the $N=2$ theory are hyper-Kähler. The classical metric is not corrected by quantum effects. Therefore the exact Higgs branch metric is obtained by the hyper-Kähler quotient construction.

In order to compare to the metric that we will compute using the fivebrane of $\mathrm{M}$ theory we have to express the metric in the coordinates of the brane construction. In order to do that we will use the following strategy. First we will consider the dimensional reduction of the $N=2$ theory to an $N=4$ theory in three dimensions. The metric on the Higgs branch is not modified under the reduction. In three dimensions there is a mirror symmetry [50] relating two different $N=4$ theories in the infrared, namely in the limit $1 / e^{2} \rightarrow 0$ where $e$ is the three dimensional gauge coupling. Under this mirror symmetry 
the Higgs and Coulomb branches of the mirror theories are exchanged. It turns out that the Higgs branch metric expressed in terms of the Coulomb branch metric of the mirror in three dimensions is naturally related to the one computed using the fivebrane. While the Higgs branch is not corrected quantum mechanically the Coulomb branch is. The metric on the Coulomb branch receives both loop and instanton corrections. In the next subsection, we will see that these have natural interpretations from the fivebrane point of view.

We will start by considering the gauge group $S U\left(N_{c}\right)$ with $N_{f}=N_{c}$ hypermultiplets in the fundamental representation. The baryonic branch of this theory has complex dimension two. The effective field theory at the baryonic branch root (the point of intersection with the Coulomb branch) is a $U(1)^{N_{c}-1}$ gauge theory with $2 N_{c}-N_{f}$ massless electrons with charges $(-1,0, \ldots, 0),(1,-1, \ldots, 0), \ldots,(0, \ldots, 1,-1),(0, \ldots, 0,1)[48]$.

The mirror theory is a $U(1)$ gauge theory with $N_{c}$ hypermultiplets (electrons) [50]. The exact Coulomb branch metric of this theory is determined at one loop. Higher loop corrections are absent [49], while instanton corrections are absent since the gauge group is abelian. The exact metric on the Coulomb branch of the mirror theory takes in the infrared limit the form

$$
d s^{2}=V(\vec{w}) d \vec{w}^{2}+V(\vec{w})^{-1}(d \sigma+\vec{A} d \vec{w})^{2}
$$

where

$$
V(\vec{w})=\frac{N_{c}}{|w|}, \quad \vec{\nabla} V=\vec{\nabla} \times \vec{A}
$$

The computation leading to (6.2) is one-loop with gauge fields on the external legs and hypermultiplets running in the loop. This is the metric of an ALE space with $A_{N_{c}-1}$ singularity.

Consider next the gauge group $S U\left(N_{c}\right)$ with $N_{f}=N_{c}+1$ hypermultiplets in the fundamental representation. The baryonic branch of this theory has complex dimension $2 N_{c}+2$. The effective field theory at the baryonic branch root is a $U(1)^{N_{c}-1}$ gauge theory 
with $2 N_{c}$ electrons charged as [48]

$\begin{array}{ccccc} & U(1)_{1} & U(1)_{2} & \ldots & U(1)_{N_{c}-1} \\ q_{1} & 1 & 1 & \ldots & 1 \\ q_{2} & 1 & 1 & \ldots & 1 \\ \vdots & & & & \\ q_{N_{c}+1} & 1 & 1 & \ldots & 1 \\ q_{N_{c}+2} & -1 & 0 & \ldots & 0 \\ q_{N_{c}+3} & 0 & -1 & \ldots & 0 \\ \vdots & \vdots & \vdots & \vdots & \vdots \\ q_{2 N_{c}} & 0 & 0 & \ldots & -1\end{array}$

The mirror theory is a $U(1)^{N_{f}-N_{c}}=U(1)^{N_{c}+1}$ gauge theory with $2 N_{c}$ electrons and charges (after a change of basis) as follows [51]

$\begin{array}{cccccc} & U(1)_{1} & U(1)_{2} & U(1)_{3} & \ldots & U(1)_{N_{c}+1} \\ q_{1} & 1 & 0 & 0 & \ldots & 0 \\ q_{2} & 1 & 0 & 0 & \ldots & 0 \\ \vdots & & & & & \\ q_{N_{c}-1} & 1 & 0 & 0 & \ldots & 0 \\ q_{N_{c}} & 1 & -1 & 0 & \ldots & 0 \\ q_{N_{c}+1} & 0 & 1 & -1 & \ldots & 0 \\ \vdots & & & & & \\ q_{2 N_{c}-1} & 0 & 0 & 0 & \ldots & -1 \\ q_{2 N_{c}} & 0 & 0 & 0 & \ldots & 1\end{array}$

As in the case $N_{f}=N_{c}$, the exact coulomb branch metric of this theory is determined at one loop. The metric takes the form [51]

$$
d s^{2}=g_{i j} d \vec{w}_{i} d \vec{w}_{j}+\left(g^{-1}\right)_{i j}\left(d \sigma_{i}+\vec{A}_{i k} d \vec{w}_{k}\right)\left(d \sigma_{j}+\vec{A}_{j l} d \vec{w}_{l}\right)
$$

where

$$
\begin{aligned}
\vec{\nabla}_{k} g_{i j} & =\vec{\nabla}_{i} g_{k j} \\
\frac{\partial}{\partial w_{i}^{m}} A_{j k}^{n}-\frac{\partial}{\partial w_{j}^{n}} A_{i k}^{m} & =\varepsilon_{m n p} \frac{\partial}{\partial w_{i}^{p}} g_{j k} .
\end{aligned}
$$

$g_{i j}$ has been computed in [51] (see eq. 4.10 there). For instance the diagonal components $g_{i i}$ take the form

$$
g_{11}=\frac{N_{c}-1}{\left|\vec{w}_{1}\right|}+\frac{1}{\left|\vec{w}_{1}-\vec{w}_{2}\right|}
$$




$$
\begin{aligned}
g_{i i} & =\frac{1}{\left|\vec{w}_{i}-\vec{w}_{i-1}\right|}+\frac{1}{\left|\vec{w}_{i+1}-\vec{w}_{i}\right|} \quad i=2, \ldots, N_{c} \\
g_{N_{c}+1, N_{c}+1} & =\frac{1}{\left|\vec{w}_{N_{c}}-\vec{w}_{N_{c}+1}\right|}+\frac{1}{\left|\vec{w}_{N_{c}}\right|}
\end{aligned}
$$

As in the previous case, there are no higher loop or instanton corrections to the metric.

The same analysis as above can be repeated for the $r=1$ non-baryonic branch for general $N_{f}$ and $N_{c}$. In this case the effective gauge theory at the root is abelian and the exact metric can be determined by one loop computation of the Coulomb branch of the mirror theory. (The theory at the root is actually the mirror of the theory at the baryonic branch root of the $N_{f}=N_{c}$ theory considered above.)

For other cases, the story is much more involved. Consider the $r>1$ non baryonic branch. The effective theory at a generic point of the root is $U(r)$ with $N_{f}$ hypermultiplets in the fundamental representation (plus a free Maxwell theory). The mirror gauge theory has gauge group $U(1) \times U(2) \times \cdots \times U(r-1) \times U(r)^{N_{f}-2 r+1} \times U(r-1) \times \cdots \times U(1)$, with hypermultiplets in the bi-fundamentals of each two adjacent groups and a hypermultiplet in the fundamental representation of each of the first and the last $U(r)[4,52]$.

Since the theory is non abelian, the one-loop metric receives contributions both from hypermultiplets and vector multiplets running in the loop. Let us label the unitary groups by $i, j, \ldots=1, \ldots, N_{f}-1$ and by $a_{i}, b_{i}, \ldots=1, \ldots, k_{i}$ the color indices of the $i$ th group $U\left(k_{i}\right)$. The Coulomb branch of the mirror theory is parametrized by $\vec{w}_{i a_{i}}$ and $\sigma_{i a_{i}}$. While the hypermultiplet in the $(i \bar{j})$ bi-fundamental contributes $1 /\left|\vec{w}_{i a_{i}}-\vec{w}_{j b_{j}}\right|$ to the $g_{i a_{i}, i a_{i}}$ component of the metric, a vector multiplet contributes $-2 /\left|\vec{w}_{i a_{i}}-\vec{w}_{i b_{i}}\right|[53]$, which becomes large negative in the limit $\vec{w}_{i a_{i}} \rightarrow \vec{w}_{i b_{i}}$. However, there are also instanton corrections relevant in this region coming from the 't Hooft-Polyakov monopole, which are typically of order

$$
\mathrm{e}^{-\frac{\left|\vec{w}_{i a_{i}}-\vec{w}_{i b_{i}}\right|}{e^{2}}}
$$

These render the full metric positive definite.

\subsection{Fivebrane Back Reaction}

In the Higgs branch, the fivebrane worldvolume has several disjoint components. In the IIA pictures, they are segments of D4 branes stretched between D6 branes and their positions parametrize the Higgs branches of the $N=2$ theory. In the M theory picture, these are fivebranes wrapping the $\mathbf{P}^{1}$ cycles on the Taub-NUT space [5,9]. The Higgs branch coordinates are then the locations (labeled by $\vec{w}$ ) of the $\mathbf{P}^{1}$ parts of the fivebrane transverse to the Taub-NUT geometry and vevs (labeled by $\sigma$ ) of the self-dual two-form $B$ 
on the $\mathbf{P}^{1}$ 's. They combine to make hyper-Kähler coordinates appropriate for the $N=2$ Higgs branch.

Let us pay attention to one of such $\mathbf{P}^{1}$ parts of the fivebrane and define

$$
\left(x^{7}, x^{8}, x^{9}\right)=\ell_{11}^{3} \vec{w}, \quad B_{z \bar{z}}=\sigma \frac{\omega_{z \bar{z}}}{\operatorname{vol}\left(\mathbf{P}^{1}\right)} .
$$

To do the Kaluza-Klein reduction on the fivebrane worldvolume, we regard $\vec{w}$ and $\sigma$ as functions of $x^{0, \ldots, 3}$ and set $\omega_{z \bar{z}}$ to be the volume form on $\mathbf{P}^{1}$. We have chosen the normalization of $\sigma$ so that its periodicity is 1 . If we use the flat metric for the spacetime, the fivebrane effective action for $\vec{w}$ and $\sigma$ would simply be

$$
\int d^{4} x\left(\operatorname{vol}\left(\mathbf{P}^{1}\right) \partial^{m} \vec{w} \partial_{m} \vec{w}+\frac{1}{\operatorname{vol}\left(\mathbf{P}^{1}\right)} \partial^{m} \sigma \partial_{m} \sigma\right),
$$

and does not correctly reproduce the Higgs branch metric expected from the field theory analysis. In the low energy approximation of $\mathrm{M}$ theory, the only way that other components of the fivebrane affect the kinetic term for the $\mathbf{P}^{1}$ is through the graviton exchange.

As a warm-up exercise, let us compute the back reaction effect when the size of $\mathbf{P}^{1}$ is larger than the distance between the branes. In this limit, the fivebrane configuration is almost flat. If a fivebrane is stretched in the $x^{0, \ldots, 3}, x^{4,5}$ directions, the metric induced by it is

$$
\begin{aligned}
d s^{2} & =g_{\mu \nu} d x^{\mu} d x^{\nu} \\
& =\left(1+\frac{\ell_{11}^{3}}{r^{3}}\right)^{-1 / 3} \sum_{i=0}^{5}\left(d x^{i}\right)^{2}+\left(1+\frac{\ell_{11}^{3}}{r^{3}}\right)^{2 / 3} \sum_{i=6}^{10}\left(d x^{i}\right)^{2},
\end{aligned}
$$

where

$$
r=\sqrt{\sum_{i=6}^{10}\left(x^{i}\right)^{2}}
$$

and the horizon of the fivebrane is located at $r=0$. We then consider another fivebrane located at $\left(x^{7}, x^{8}, x^{9}\right)=\ell_{11}^{3} \vec{w}$ and stretched in the $x^{0, \ldots, 3}, x^{6,10}$ directions. To derive the kinetic term for $\vec{w}$, we take $\vec{w}$ to be a function of $\left(x^{0}, \ldots, x^{3}\right)$ and substitute it into the fivebrane action:

$$
S_{\vec{w}}=\int d x^{0} \cdots d x^{3} \int d x^{6} d x^{10} \sqrt{\operatorname{det} G} \sum_{m, n=0}^{3} \sum_{a, b=7,8,9} G^{m n} g_{a b} \partial_{m} w^{a} \partial_{n} w^{b},
$$

where $g_{a b}$ is the spacetime metric given by (6.11) and $G_{m n}$ is the induced metric on the fivebrane. This integral can be evaluated explicitly and one finds,

$$
S_{\vec{w}}=\int d^{4} x \int d x^{6} d x^{10}\left(1+\frac{\ell_{11}^{3}}{\left(\sum_{i=6}^{10}\left(x^{i}\right)^{2}\right)^{3 / 2}}\right) \partial^{m} \vec{w} \cdot \partial_{m} \vec{w}
$$




$$
=\int d^{4} x\left(\operatorname{vol}\left(\mathbf{P}^{1}\right)+\frac{\pi / 2}{|w|}\right) \partial^{m} \vec{w} \cdot \partial_{m} \vec{w}
$$

Note that the $\ell_{11}$ dependence disappears from the final expression.

The kinetic term for $\sigma$ comes from

$$
S_{B}=\int d^{6} x\left|d B-C^{(3)}\right|^{2}
$$

of the fivebrane action, so we have to evaluate the 3 -form potential $C^{(3)}$ induced by the fivebrane at $r=0$, i.e.

$$
d C^{(3)}=\frac{1}{r^{5}} \sum_{i j k l m=6}^{10} x^{i} d x^{j} \wedge d x^{k} \wedge d x^{l} \wedge d x^{m}
$$

Up to a suitable gauge choice, we can solve this as

$$
\begin{aligned}
C_{a=7,8,9 ; i j=6,10}^{(3)} & =A_{a} \frac{y^{3}}{r^{5}} \epsilon_{i j} \\
C_{a, b=7,8,9 ; i=6,10}^{(3)} & =x_{\left[a, A_{b]}\right.} \frac{1}{r^{5}} \epsilon_{i j} x^{j} \\
C_{789}^{(3)} & =0,
\end{aligned}
$$

where $A_{i}$ is the magnetic monopole vector potential in the $x^{7,8,9}$ space,

$$
A_{i} d x^{i}=(\cos \theta \pm 1) d \phi
$$

and $y=\sqrt{\sum_{a=7,8,9}\left(x^{a}\right)^{2}}$. On $\mathbf{P}^{1}$ where the fivebrane is wrapped, $A_{a}$ is constant and $y^{3} / r^{5}$ is proportional to the volume form $\omega_{z \bar{z}}$ up to a cohomologically trivial form. Thus, with a suitable shift of the 2 -form $B$ in the definition of $\sigma$ in (6.9), we find

$$
\left(d B-C^{(3)}\right)_{\mid \mathbf{R}^{4} \times \mathbf{P}^{1}}=\left(d \sigma-A_{i} d x^{i}\right)_{\mid \mathbf{R}^{4}} \wedge \frac{\omega_{z \bar{z}}}{\operatorname{vol}\left(\mathbf{P}^{1}\right)}
$$

Notice that when we consider $d B-C^{(3)}$ on $\mathbf{R}^{4} \times \mathbf{P}^{1}$, there are also terms coming from the second line in (6.17), but these give rise to higher derivative terms in the action and have been dropped in (6.19). The three-form in (6.19) is not yet self-dual. To make it self-dual, we add to $B$ the two-form

$$
B_{0}=\left(1+\frac{1}{r^{3}}\right)^{-1} \frac{1}{\operatorname{vol}\left(\mathbf{P}^{1}\right)} x_{n}\left(\partial_{m} \sigma-A_{i} \partial_{m} x^{i}\right)\left(d x^{n} \wedge d x^{m}+\epsilon_{p q}^{n m} d x^{p} \wedge d x^{q}\right)
$$

where $m, n, p, q \in\{0,1,2,3\}$. Again dropping higher derivative terms, $d B_{0}$ contains two pieces. One of them is the dual of (6.19), the other is a self-dual three-form. Thus, $d\left(B+B_{0}\right)-C^{(3)}$ is a self-dual three-form. In addition, the gauge fixing conditions $d^{*} B=0$ 
and $d^{*} C^{(3)}=0$ are satisfied. This is precisely the situation as described in section 2 , showing that we should do our computations with the three-form $d\left(B+B_{0}\right)-C^{(3)}$. Luckily, $\int\left|d\left(B+B_{0}\right)-C^{(3)}\right|^{2}=2 \int\left|d B-C^{(3)}\right|^{2}$, so we do not make an error is we work with just the three-form (6.19).

The action is then evaluated as

$$
\begin{aligned}
S_{\sigma} & =\int d^{4} x \int \frac{d x^{6} d x^{10}}{\left(\operatorname{vol}\left(\mathbf{P}^{1}\right)\right)^{2}}\left(1+\frac{\ell_{11}^{3}}{\left(\sum_{i=6}^{10}\left(x^{i}\right)^{2}\right)^{3 / 2}}\right)^{-1}\left(\partial_{m} \sigma-A_{i}(w) \partial_{m} w^{i}\right)^{2} \\
& =\int d^{4} x\left(\frac{1}{\operatorname{vol}\left(\mathbf{P}^{1}\right)}-\frac{\pi / 2}{\left(\operatorname{vol}\left(\mathbf{P}^{1}\right)\right)^{2}|w|}+\cdots\right)\left(\partial_{m} \sigma-A_{i}(w) \partial_{m} w^{i}\right)^{2}
\end{aligned}
$$

Combining this with (6.14), we find that the metric on the moduli space of the fivebrane wrapping on $\mathbf{P}^{1}$ is given by

$$
d s^{2}=V(w) d \vec{w}^{2}+V(w)^{-1}\left(d \sigma-A_{i} d w^{i}\right)^{2}
$$

with

$$
V(w)=\operatorname{vol}\left(\mathbf{P}^{1}\right)+\frac{\pi / 2}{|w|}
$$

and

$$
\nabla V=\nabla \times A
$$

Although we have obtained (6.23) by computing each term explicitly, we could have gotten it faster by using the following argument. It is known that (6.23) with $V$ and $A$ obeying (6.25) is the most general hyper-Kähler metric for $(\sigma, \vec{w})$ with the rotational symmetry for $\vec{w}$ and the translational symmetry for $\sigma$. The condition (6.25) requires $\Delta V=0$ almost everywhere except for singularities in $V$. In the present case, a singularity comes only from $\vec{w}=0$. The vector potential $A$ is then uniquely determined by the intersection of the two components of the fivebranes, one stretching in the $x^{4,5}$ directions and the other stretching in the $x^{6,10}$ directions. This in turn fixes $V$ up to an additive constant.

This argument is also applicable when the size of $\mathbf{P}^{1}$ is comparable to $x^{7,8,9}$ or smaller. In this case, we can no longer ignore the effect of the background Taub-NUT geometry. The metric induced by the fivebrane wrapping on cycles on the Taub-NUT space is not known explicitly. Nevertheless it is still true that the resulting moduli space metric in the supergravity limit should be of the form (6.23) with $V$ and $A$ obeying (6.25). This is because the rotational symmetry in the $x^{7,8,9}$ plane is unaffected by the Taub-NUT geometry and the translational invariance in $\sigma$ is unbroken in the supergravity limit. It is then sufficient to determine $A$, which can be found as follows. 
Suppose the fivebrane is wrapped on a surface $\mathbf{R}^{4} \times \Sigma$ at the origin of the $x^{7,8,9}$ plane, where $\Sigma$ is a cycle on the Taub-NUT space. Let $C^{(3)}$ be the 3 -form potential induced by such a fivebrane. Then for any other cycle $\Sigma^{\prime}$ on the Taub-NUT space,

$$
\int_{S^{2} \times \Sigma^{\prime}} d C^{(3)}=\#\left(\Sigma \cap \Sigma^{\prime}\right)
$$

where $S^{2}$ surround the origin in the $x^{7,8,9}$ plane and $\#\left(\Sigma \cap \Sigma^{\prime}\right)$ is the intersection number of the two surfaces. This means

$$
C^{(3)}=\frac{\#\left(\Sigma \cap \Sigma^{\prime}\right)}{\operatorname{vol}\left(\Sigma^{\prime}\right)} A_{i} d x^{i} \wedge \omega_{z \bar{z}}+\cdots
$$

where $A_{i}$ is the vector potential for the monopole at the origin of the $x^{7,8,9}$ plane and $\omega_{z \bar{z}}$ is the volume form on $\Sigma^{\prime}$. This then determines $A$ for the moduli space metric.

It is straightforward to generalize this construction when there are several disjoint $\mathbf{P}^{1}$ 's. In this case, the metric takes the form (cf. (6.5) and (6.6))

$$
d s^{2}=g_{i j} d \vec{w}_{i} d \vec{w}_{j}+\left(g^{-1}\right)_{i j}\left(d \sigma_{i}+\vec{A}_{i k} d \vec{w}_{k}\right)\left(d \sigma_{j}+\vec{A}_{j l} d \vec{w}_{l}\right),
$$

where

$$
\begin{aligned}
\vec{\nabla}_{k} g_{i j} & =\vec{\nabla}_{i} g_{k j} \\
\frac{\partial}{\partial w_{i}^{a}} A_{j k}^{b}-\frac{\partial}{\partial w_{j}^{b}} A_{i k}^{a} & =\varepsilon_{a b c} \frac{\partial}{\partial w_{i}^{c}} g_{j k} .
\end{aligned}
$$

Here $\vec{w}_{i}$ specifies the location of the $i$-th $\mathbf{P}^{1}$ and $\sigma_{i}$ is the vev of the self-dual $B$-field on it. By generalizing the above argument, one can show that $A_{i j}$ is uniquely determined by the intersection of the $i$-th and the $j$-th $\mathbf{P}^{1}$. In the Taub-NUT space of $A_{N}$ type, the intersection number of neighboring cycles on the Taub-NUT space is +1 while the self-intersection number of each cycle is -2 . Thus, with the identification $1 / e^{2}=\operatorname{vol}\left(\mathbf{P}^{1}\right)$, one can easily see that the metric computed in this way agrees with the field theory result in the previous subsection, up to the instanton corrections.

To obtain the four-dimensional gauge theory from the fivebrane, we should be able to neglect the Kaluza-Klein modes. Since the typical energy scale of the gauge theory is $|w|$, we need

$$
|w| \ll \frac{1}{\operatorname{vol}\left(\mathbf{P}^{1}\right)} .
$$

This is the same as taking the infrared limit $1 / e^{2} \rightarrow 0$ in the field theory analysis in the previous subsection. This is also the limit where the $N_{f}$ D6-branes, separated in the $x^{6}$ direction, become close to one another and the $S U\left(N_{f}\right)$ symmetry is restored. This is 
indeed the limit where the worldvolume theory becomes close to the gauge theory since the latter has the $S U\left(N_{f}\right)$ flavor symmetry. In the spacetime coordinates related to $\vec{w}$ by (6.9), (6.30) means

$$
\left|x^{7,8,9}\right| \operatorname{vol}\left(\mathbf{P}^{1}\right) \ll \ell_{11}^{3} .
$$

One can expect that quantum corrections to the supergravity description become strong in such a situation. ${ }^{1}$ To see this more explicitly, let us consider the case when there are two components of the fivebrane wrapping on two copies of a $\mathbf{P}^{1}$ of the TaubNUT space separated in the $x^{7,8,9}$ directions. As a membrane can end on the fivebrane, a membrane stretched between the two $\mathbf{P}^{1}$ may cause instanton effects. The membrane action measured with respect to the metric (6.11) would be proportional to $\operatorname{vol}\left(\mathbf{P}^{1}\right)\left|\Delta x^{7,8,9}\right| / \ell_{11}^{3}=\operatorname{vol}\left(\mathbf{P}^{1}\right)|\Delta \vec{w}|$ and thus the instanton effects are typically

$$
\mathrm{e}^{-\operatorname{vol}\left(\mathbf{P}^{1}\right)|\Delta \vec{w}|}
$$

which become large in the limit (6.30) or (6.31). The instanton would break the translational invariance in $\sigma$, and thus the statement in the above paragraph does not hold in such a case. By following a chain of duality arguments, one can show that these membrane instantons are in one-to-one correspondence to the field theory instantons discussed in the previous subsection and have the same effect on the metric (compare (6.32) with $(6.8))$.

So far we have been considering the variation of the $x^{7,8,9}$ position and the chiral two form of the finite $\mathbf{P}^{1}$ components. We have shown that the brane computation correctly reproduces the field theory result for non-baryonic branches (up to the membrane instanton correction). However, for the baryonic branches, there is one quaternionic modulus in addition to such $\mathbf{P}^{1}$ motions. As discussed in [9], this is identified with the one modulus associated with the charged massless particles which appear when the infinite component factorizes into two components. It is natural to guess that this is related to the relative separation of the two components. However, since both components are of infinite volume, a naïve computation shows that it costs an infinite energy to separate them and also it is not clear whether there is a zero mode for the chiral two form. Nevertheless, if we assume that there is one zero mode for the chiral two form and there is a way to make the kinetic energy finite, the back reaction method again gives us the correct field theory result. Note that the two infinite components intersect at $2 N_{c}-N_{f}$ points [9]. In

\footnotetext{
${ }^{1}$ It was shown in [54] that the fivebrane metric (6.11) is smooth across the horizon at $r=0$. This however is not the case when the fivebrane is wrapped on a compact surface such as $\mathbf{P}^{1}$ and the curvature is expected to diverge near the horizon. We thank G. Horowitz and J. Maldacena for discussion on this issue.
} 
the case $N_{f}=N_{c}$, this yields the equation $V=N_{c} /|\vec{w}|$ in (6.2). In the case $N_{f}=N_{c}+1$, $\vec{w}_{1}$ is interpreted as the location of one of the infinite components and the other is fixed at $\vec{w}=0$. Then, the first and the last equation in (6.7) follow from the fact that the two infinite components intersect at $N_{c}-1$ points, and that one of them intersects the first $\mathbf{P}^{1}$ at one point whereas the other intersects the last $\mathbf{P}^{1}$ at one point.

Remarks.

(1) The Higgs branch metric has also been discussed in the framework of geometric engineering in [55] where the Higgs branch of an $U(1)$ theory was considered.

(2) We also note that the above computation can be extended to $S p\left(N_{c}\right)$ gauge theories and compactified $6 \mathrm{~d}$ tensionless string theory by using the configuration constructed in [52]. In these cases, open membrane instantons always contribute.

\section{$7 \quad N=1$ Higgs Branch}

We consider four-dimensional $N=1$ supersymmetric gauge theory in the phase where the gauge group is broken by the Higgs mechanism. We consider two kinds of theories. One is obtained from $N=2 \mathrm{SQCD}$ with $S U\left(N_{c}\right)$ group and $N_{f}$ flavors by giving mass $\mu$ to the adjoint chiral multiplet. The other is $N=1$ supersymmetric QCD. The fivebrane configuration in $M$ theory corresponding to these theories has been obtained in [9]. We consider the simplest example $N_{c}=N_{f}=2$ in some detail.

In both cases, we use the back-reaction method to compute the metric on the moduli space as in the computation of the metric of the $N=2$ Higgs branch. In the case of $N_{f}=N_{c}=2$, there are no complications associated with open membrane instantons.

\section{1 $N=2$ Broken to $N=1$ by Adjoint Mass}

\section{Field Theory}

$N=2$ gauge theory with $S U(2)$ gauge group and $N_{f}=2$ fundamental hypermultiplets has been studied in detail in [6]. The moduli space consists of a complex one-dimensional Coulomb branch and two Higgs branches emanating from different points on the Coulomb branch. One of the Higgs branches is of baryonic type and the other is of non-baryonic type. These are both isomorphic to an Eguchi-Hanson space with an $A_{1}$ singularity at the point of intersection with the Coulomb branch.

If we give a bare mass $\mu$ to the adjoint chiral multiplet, $N=2$ supersymmetry is 
broken to $N=1$ and all but the two Higgs branches are lifted. The Higgs branch is Kähler but no longer a hyper-Kähler manifold, and it is difficult to compute the Kähler metric because it received both loop and instanton corrections. However, it is easy to see how the complex structure of these Higgs branches is deformed as a function of $\mu$. Here we only consider the non-baryonic branch. The non-baryonic branch is parametrized by the meson vev $M=\widetilde{Q} Q$ which is a $2 \times 2$ complex matrix. As analyzed in [9], a general meson vev can be made by a flavor rotation into a diagonal matrix with eigenvalues $(i \mu \Lambda,-i \mu \Lambda)$ where $\Lambda$ is the dynamical scale of the $N=2$ theory (therefore the moduli space is set-theoretically the homogeneous space $\left.S L(2, \mathbf{C}) / \mathbf{C}^{*}\right)$. Thus, at a general point of the moduli space, the meson matrix is expressed as

$$
M=g\left(\begin{array}{cc}
i \mu \Lambda & 0 \\
0 & -i \mu \Lambda
\end{array}\right) g^{-1}=i \mu \Lambda\left(\begin{array}{cc}
a d+b c & 2 a b \\
2 c d & -a d-b c
\end{array}\right), \quad a d-b c=1 .
$$

In terms of the variables $M_{11}=W, M_{12}=X, M_{21}=Y$, the moduli space is described as a complex manifold by

$$
X Y=(W-i \mu \Lambda)(W+i \mu \Lambda) .
$$

Namely, the $A_{1}$ singularity of the $\mu=0$ Higgs branch has split into two $A_{0}$ "singularities" and is smoothed out. Although this complex manifold admits a hyper-Kähler metric, we do not expect the metric of the moduli space to be hyper-Kähler.

\section{Fivebrane Back Reaction}

In [9], we have seen that the fivebrane correctly reproduces the relation of the flavor invariants of supersymmetric QCD with and without heavy adjoint. There we also proposed that the flavor rotation is related to the position of the $\mathrm{CP}^{1}$ components by counting the number of degrees of freedom. However, the precise map between the position of the $\mathbf{C P}^{1}$ components and the points in the flavor orbit has not been given. Here we compute the metric of the non-baryonic branch by the back-reaction method and read off its complex structure to compare with (7.2).

As shown in $[5,9]$, the fivebrane at this non-baryonic branch consists of two components - an infinite component $C$ and a finite component $\mathbf{P}^{1}$. The position $\vec{w}$ of the $\mathbf{P}^{1}$ component in the 789 directions and the integral $\sigma$ of the chiral two form on $\mathbf{P}^{1}$ correspond to the real four dimensions of this branch. For $\mu=0$, the $\mathbf{P}^{1}$ component intersects the infinite component $C$ at two points at the origin $\vec{w}=0$ of the 789 direction [5]. For $\mu \neq 0$, the infinite component $C$ is rotated in the $45-89$ plane, and the $\mathbf{P}^{1}$ component intersects $C$ at one point at one value of $\vec{w},\left(w^{7}, w=w^{8}+i w^{9}\right)=(0, i \mu \Lambda)$, and at one point at another value $\left(w^{7}, w\right)=(0,-i \mu \Lambda)[9]$ (see Figure 2). 


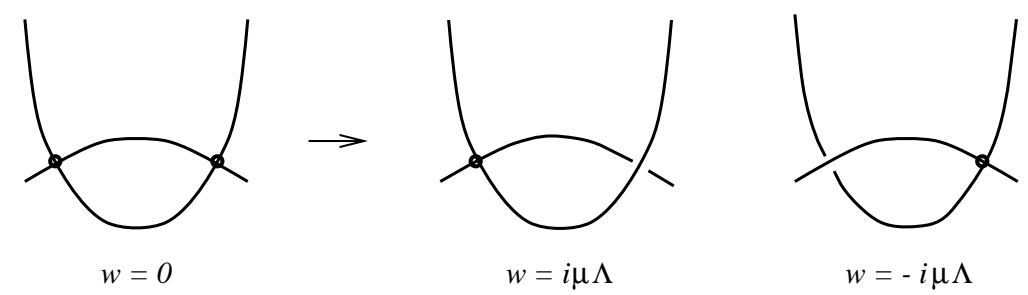

Figure 2: Splitting $A_{1}$ Singularity to $A_{0} \times A_{0}$ by Adjoint Mass

The motion of the $\mathbf{P}^{1}$ component is affected by the infinite component $C$ and the effect on the metric is localized at these intersection points when all characteristic scales are much larger than the eleven-dimensional Planck scale. As shown in the previous section, the metric for $\mu=0$ is given by

$$
\mathrm{d} s^{2}=U(\mathrm{~d} \vec{w})^{2}+U^{-1}(\mathrm{~d} \sigma+\vec{A} \cdot \mathrm{d} \vec{w})^{2}
$$

where

$$
\begin{aligned}
& \operatorname{curl} \vec{A}=\operatorname{grad} U \\
& U=\operatorname{vol}\left(\mathbf{P}^{1}\right)+\frac{2}{|\vec{w}|}
\end{aligned}
$$

The numerator " 2 " in the expression for $U$ shows that the $\mathbf{P}^{1}$ component intersects $C$ at two points at the same time, and indicates the $A_{1}$ singularity.

In the case of $\mu \neq 0$, the two intersection points split into one intersection point at $w=i \mu \Lambda$ and one intersection point at $w=-i \mu \Lambda$ (both at $w^{7}=0$ ). Moreover, the infinite component $C$ is sloped in the $v-w$ directions as:

$$
w \sim \pm \frac{\mu}{2} v
$$

at the intersection point $w= \pm i \mu \Lambda$. Then, a naïve back-reaction method would show that the metric of this branch is given by (7.3) in which curl $\vec{A}=\operatorname{grad} U$ and $U$ is given by

$$
U=\operatorname{vol}\left(\mathbf{P}^{1}\right)+\frac{1}{\sqrt{c^{2}|w-i \mu \Lambda|^{2}+\left(w^{7}\right)^{2}}}+\frac{1}{\sqrt{c^{2}|w+i \mu \Lambda|^{2}+\left(w^{7}\right)^{2}}}
$$

where $c^{2}$ is a constant of order $\sim 1 /\left(1+|R \mu / 2|^{2}\right)$. This is only an approximation since there is no reason why the coefficients of $(\mathrm{d} \vec{w})^{2}$ and $(\mathrm{d} \sigma+\cdots)^{2}$ should be the same in the case where $N=2$ supersymmetry is broken to $N=1$. However, not knowing the correct way to carry out the computation, we examine this naïve approximation of the metric. 
There is a complex structure with respect to which this metric is hermitian. One of the complex coordinates is $w$. Another coordinate is given by

$$
y=\mathrm{e}^{-\left(\operatorname{vol}\left(\mathbf{P}^{1}\right) w^{7}+i \sigma\right)} \prod_{\epsilon= \pm i} \sqrt{\sqrt{c^{2}|w-\epsilon \mu \Lambda|^{2}+\left(w^{7}\right)^{2}}-w^{7}} \cdot \text { const. }
$$

Then, the metric is expressed as

$$
\mathrm{d} s^{2}=U|\mathrm{~d} w|^{2}+U^{-1}|\mathrm{~d} y / y-\delta \mathrm{d} w|^{2},
$$

where

$$
\delta=\frac{1}{2} \sum_{\epsilon= \pm i} \frac{1}{w-\epsilon \mu \Lambda}\left(1+\frac{w^{7}}{\sqrt{c^{2}|w-\epsilon \mu \Lambda|^{2}+\left(w^{7}\right)^{2}}}\right) .
$$

Thus, the metric is indeed hermitian. There is yet another complex coordinate

$$
x=\mathrm{e}^{\operatorname{vol}\left(\mathbf{P}^{1}\right) w^{7}+i \sigma} \prod_{\epsilon= \pm i} \sqrt{\sqrt{c^{2}|w-\epsilon \mu \Lambda|^{2}+\left(w^{7}\right)^{2}}+w^{7}} \frac{w-\epsilon \mu \Lambda}{|w-\epsilon \mu \Lambda|} \cdot \text { const. }
$$

Then, $x, y$ and $w$ satisfy the relation

$$
x y=\text { const } \cdot(w-i \mu \Lambda)(w+i \mu \Lambda),
$$

and this describes the complex structure of the moduli space with respect to which the metric is hermitian. This agrees with the field theory result (7.2). Thus, we propose that the $\mathbf{P}^{1}$ motion is mapped to the flavor rotation by $x=X, y=Y$, and $w=W$.

However, there is a serious problem. Since the theory is $N=1$ supersymmetric, the moduli space of vacua must be Kähler. Although the above metric is hermitian, it is not Kähler with respect to the above chosen complex structure. It is not clear whether this is because of the too naïve approximation or because of the wrong choice of the complex structure. We leave it as an open problem. If we can show that the reason is the former but not the latter, it would support the above refinement of the proposal about the relation of the flavor rotation and the $\mathbf{P}^{1}$ motion.

In principle, the correct complex structure could be identified by looking at the action of the supersymmetry on the worldvolume fields or on the parameters $(\vec{w}, \sigma)$. Although it is not clear whether it is practical, it is important to show that the complex structure of the moduli space is independent of the extra parameter $R$. It is clear that the part of the complex structure which is directly induced from the complex structure of the space-time (such as $w=w^{8}+i w^{9}$ ) does not depend on the extra parameter $R$, but it is less clear whether it is true for the part related to parameters such as $\left(w^{7}, \sigma\right){ }^{1}$

\footnotetext{
${ }^{1}$ It is interesting to note that the pair $\left(w^{7}, \sigma\right)$ looks very much like the scalar fields one gets after dualizing the linear multiplet of $N=2$ theory in three dimensions. The expression (7.8) or (7.11) is almost the same as the expression for the superpotential (which is a holomorphic function) that appeared in the study of three-dimensional $N=2$ gauge theory (see (3.11) in [56]).
} 


\section{$7.2 \quad N=1$ SQCD}

We will consider four dimensional $N=1$ supersymmetric gauge theory with gauge group $S U(2)$ and $N_{f}=2$ pairs of chiral multiplets in the fundamental and anti-fundamental representations. The moduli space of vacua is a Kähler manifold whose complex structure is corrected by a non-perturbative effect. The classical metric is corrected by quantum effects both perturbatively and non-perturbatively. As in the $N=2$ case, the comparison to the brane computation becomes more transparent if we express the metric by compactifying on a circle to three dimensions and go to the mirror gauge theory. The compactification on a circle of an $N=1$ theory in four dimensions is a three-dimensional $N=2$ theory. The classical metric on the Higgs branch of the four dimensional $N=1$ theory is identical to the classical metric of the three dimensional $N=2$ theory. In a certain class of three-dimensional $N=2$ theories, there is a mirror symmetry relating two different theories in the infrared [57], namely in the limit $1 / e^{2} \rightarrow 0$ where $e$ is the three dimensional gauge coupling. Under this mirror symmetry the Higgs and Coulomb branches of the mirror theories are exchanged. Although both the Higgs and Coulomb branches get corrected quantum mechanically, the one loop correction to the Coulomb branch metric is seen classically on the Higgs branch of the mirror. We will show that the metric obtained using the back reaction method in the previous section is close to the one loop metric on the Coulomb branch of the mirror theory. Instanton corrections to the Coulomb branch metric correspond to the membranes wrapping the three cycles which we discussed $i$ in the previous section.

The Higgs branch of this theory has complex dimension five. The M theory description of the Higgs branch was studied in [9]. There are two complex moduli $(\vec{w}, \sigma)$ associated with the motion of the $P^{1}$ in $\left(x^{7}, x^{8}, x^{9}\right)$ and the integral of the chiral 2-form on the $P^{1}$. This corresponds to the motion of a D4 brane broken between two D6 branes and the $A_{6}$ gauge field component on the D4 brane in the Type IIA picture. Two complex moduli $m_{1}, m_{2}$ are associated with the deformation of an infinite component $C_{L}$ of the curve in $\left(x^{8}, x^{9}\right)$. These correspond to the motion of two D4 branes broken between a D6 brane and the NS' brane in the Type IIA picture. One complex modulus $n$ has its real part associated with the relative motion in $x^{7}$ of the infinite components of the curve $C_{L}$ and $C_{R}$, and its imaginary part is associated to the integral of the chiral 2-form on $C_{L}$ and $C_{R}$. The real part of $n$ corresponds in the Type IIA description to a relative motion of the NS and NS' branes in $x^{7}$. A computation as in [5] implies that an infinite energy is needed for the motions $n$ and $m_{1}+m_{2}$. By considering them as moduli we are assuming that a more elaborate computation will show that only finite energies are needed for them. 
The mirror theory is a $U(1)$ gauge theory with matter content of $N=4$ gauge theory with two charged hypermultiplets and a meson $M$ that couples to the charged matter as $M_{i} \tilde{q}_{i} q_{i} \cdot{ }^{1}(\vec{w}, \sigma)$ are the vev's for the three real scalars in the vector multiplet and the dual to the gauge field respectively. In the three dimensional gauge theory $n$ is a parameter rather than a modulus, its real part is the FI parameter. Following [57] the one loop metric on the Coulomb branch of the mirror theory takes the form

$$
\begin{aligned}
d s^{2}=\left(\frac{1}{\left|\vec{w}+\vec{m}_{1}\right|}+\frac{1}{\left|\vec{w}+\vec{m}_{2}\right|}\right) d \vec{w}^{2} & +\left(\frac{1}{\left|\vec{w}+\vec{m}_{1}\right|}+\frac{1}{\left|\vec{w}+\vec{m}_{2}\right|}\right)^{-1}(d \sigma+\cdots)^{2} \\
& +\frac{1}{\left|\vec{w}+\vec{m}_{1}\right|} d m_{1} d \bar{m}_{1}+\frac{1}{\left|\vec{w}+\vec{m}_{2}\right|} d m_{2} d \bar{m}_{2},
\end{aligned}
$$

where $\vec{m}_{i}=\left(0, m_{i}\right)$. The term $\left(\frac{1}{\left|\vec{w}+\vec{m}_{1}\right|}+\frac{1}{\left|\vec{w}+\vec{m}_{2}\right|}\right)$ is a consequence of the fact that in the mirror theory the moduli $m_{1}, m_{2}$ correspond to a meson that couples to the charged hypermultiplet as $m_{i} \tilde{q}_{i} q_{i}$. The "..." part depends on $d \vec{w}, d m_{1}, d m_{2}$ and is determined by the duality transformation of the vector to a scalar in the presence of the meson, such that the metric (7.13) is Kähler. As we noted previously, the four dimensional modulus $n$ is a parameter in three dimensions.

The metric (7.13) is obtained via an approximate back reaction, where the infinite component $C_{L}$ is approximated by a straight brane and the back reaction of the infinite component $C_{R}$ is neglected. Therefore this classical supergravity approximate back reaction reproduces the one loop metric of the Coulomb branch of the mirror theory. This however only captures classical features of the metric on the Higgs branch of the original theory. We expect that the classical metric will be corrected by loops. These higher loops cannot be seen in the appproximate back reaction that we used. It is probable that an improved back reaction method that takes into account the structure of the infinite component $C_{L}$ as well as $C_{R}$ will capture the higher loops.

The example above can be generalized in a straightforward way to general $N_{f}, N_{c}$. The modifications compared to the $N=2$ case are the meson moduli that add mass terms for some of the quarks [57]. However, in this case we also expect instanton corrections and we have to compute the membrane instanton contribution.

\footnotetext{
${ }^{1}$ This mirror symmetry is approximate and it only captures a tree level coupling of the meson to the quarks and therefore cannot be used to get an exact metric on the moduli space of vacua.
} 


\section{Discussion}

The worldvolume theory on the $M$ theory fivebrane wrapped on a non-compact Riemann surface depends on a scale, the radius $R$ of the circle in the eleventh direction, in addition to the parameters that can be identified with the parameters or the moduli of the four-dimensional gauge theory. It is the presence of this additional parameter that simplifies the analysis.

In general, the worldvolume theory becomes close to a standard four-dimensional gauge theory in the region where $R \ll \ell_{11}$ and some of the characteristic lengths of the brane become small compared to $\ell_{11}$ because the degrees of freedom absent in the fourdimensional theory becomes heavy. On the other hand, $M$ theory is well-approximated by the eleven-dimensional supergravity in the opposite region where $R \gg \ell_{11}$ and all the characteristic lengths of the brane becomes much larger than $\ell_{11}$.

However, some quantities do not depend on the additional scale $R$ and can be computed by going to the region of the parameter space where the eleven-dimensional supergravity approximation is valid. As has been shown already, examples of such quantities are some flavor invariant combination of the vev of gauge invariant chiral operators, the mass of BPS particles and the tension of BPS domain walls. It seems that all the BPS or holomorphic quantities fall into this class. It is important to understand whether and why this assertion holds, and whether any exceptions can be found.

In this paper, we have computed the low energy effective action of the worldvolume theory in the region of the parameter space where the supergravity approximation is valid. In particular, we have computed Kähler metric of some $N=2$ and $N=1$ theories and higher derivative terms of $N=2$ Coulomb branch. In the case of the $N=2$ Coulomb branch metric, the effective gauge coupling of $N=1$ and $N=2$ abelian Coulomb phase, and the Higgs branch metric of some $N=2$ theories, the supergravity computation has lead to a correct result of the corresponding four-dimensional gauge theory. In all these successful cases, the computation involves the chiral two form on the fivebrane, directly or indirectly via supersymmetry, where its lagrangian in the supergravity limit is given by the second term of (2.1). This indicates that this part of the worldvolume theory of fivebrane has a remarkable property that the supergravity approximation is valid in a wider region of the parameter space than naïvely expected.

This does not apply to the calculation of non-holomorphic quantities such as the Kähler

potential of $N=1$ supersymmetric gauge theories or the higher derivative terms in the effective action of $N=2$ theories, where the computation is disconnected from the chiral 
two-form piece. We found that the results are quantitatively strikingly different than what we expect for the four dimensional gauge theories. They share however some qualitative features such as the fact that the four derivative terms in the $N=2$ effective action derived from the fivebrane theory are singular precisely at the same points in the moduli space of vacua where the four derivative terms of the four dimensional gauge theories are. It seems therefore that although the eleven-dimensional supergravity limit cannot provide a quantitative agreement between the fivebrane theory and the gauge theory, it can still be useful to extract qualitative results.

In order to use the fivebrane of $M$ theory to study four-dimensional gauge theory in detail, it seems that we need to get a better understanding of the fivebrane theory when $R$ is small and the Type IIA string theory is weakly coupled. Such an understanding might be provided by the matrix theory description of $\mathrm{M}$ theory. There are of course several difficulties in that direction. We are interested in theories with four and eight supercharges and the corresponding matrix descriptions have half of these amounts of supersymmetry. This will make the analysis of the quantum corrections difficult. Also, the realization of the brane configurations in matrix theory is not straightforward since, for instance, we do not know how to describe a transversal fivebrane in matrix theory.

Another curious point that demonstrates the need for a better understanding of the fivebrane theory is the decoupling argument. Generally in supersymmetric gauge theories we can think about parameters of the theory as vev's of background chiral superfields. This is useful in order to get a control on quantum corrections. For instance, consider a Type IIA string theory compactified on a Calabi-Yau 3-fold which leads to $N=2$ theory in four dimensions. Using the way the dilaton enters the low-energy effective action and the decoupling between the $N=2$ vector multiplet and hypermultiplets, one can show that the structure of the vector multiplet moduli space does not receive stringy corrections and can be easily computed. On the other hand the hypermultiplet moduli space does receive stringy corrections. In our case we have one additional parameter $R$. However, it is not clear in which multiplet $R$ sits. In particular, we were able to use the fivebrane theory to compute both the Coulomb and Higgs branch metric which implies that our understanding of the decoupling argument in the Type IIA compactification does not apply in an obvious way to the fivebrane theory.

\section{Acknowledgements}

We would like to thank G. Horowitz, J. Maldacena, H. Murayama and C. Vafa for useful discussions. This research is supported in part by NSF grant PHY-95-14797 and DOE grant DE-AC03-76SF00098. JdB is a fellow of the Miller Institute for Basic Research 
in Science. 


\section{Appendix}

\section{A The Space-time Metric}

We identify parameters defining the branes with some physical quantities. Specifically, in the $N=2$ configuration, we identify the position of the D4 branes in the 4,5 directions (parametrized by $v$ ) as the VEV of the scalar component of the vector multiplet $\Phi$, while the position in the $7,8,9$ directions (parametrized by $\vec{w}=\left(w^{7}, w^{8}, w^{9}\right)$ ) as the meson VEVs $|Q|^{2}-|\widetilde{Q}|^{2}, \widetilde{Q} Q$. The purpose of this note is to write down the metric of the eleven-dimensional space-time in terms of these coordinates $v, \vec{w}$. In the flat back-ground, we claim that it is given by

$$
\mathrm{d} s^{2}=-\left(\mathrm{d} x^{0}\right)^{2}+\left(\mathrm{d} x^{1}\right)^{2}+\left(\mathrm{d} x^{2}\right)^{2}+\left(\mathrm{d} x^{3}\right)^{2}+\left|\ell_{s t}{ }^{2} \mathrm{~d} v\right|^{2}+\left|\ell_{11}{ }^{3} \mathrm{~d} \vec{w}\right|^{2}+\left|R \frac{\mathrm{d} t}{t}\right|^{2}
$$

where $t$ is the complex coordinate for the 6,10 directions given by $t=\exp \left(-x^{6} / R-i x^{10}\right)$ $\left(x^{10}\right.$ has period $\left.2 \pi\right)$. In the above expression, $\ell_{s t}$ is the string length, $\ell_{11}$ is the elevendimensional Planck length, and $R$ is the radius of the circle in the eleventh direction which are related by $\ell_{11}{ }^{3}=R \ell_{s t}{ }^{2}$. In the presence of $\mathrm{D} 6$ branes at points in the 4,5,6 directions, the metric is obtained by replacing $\left|\ell_{s t}{ }^{2} \mathrm{~d} v\right|^{2}+|R \mathrm{~d} t / t|^{2}$ in (A.1) by the multi-Taub-NUT metric $\mathrm{d} s_{\mathrm{TN}}^{2}$ which is exhibited in some detail in Appendix B.

It is easy to see that the length of an interval in the $v$ direction is given by $\left|\ell_{s t}{ }^{2} \Delta v\right|$. Look at two $\mathrm{D} 4$ branes at $v=a_{i}$ and $v=a_{j}$ which are stretched between NS 5 -branes. The string stretched between these D4 branes creates the $(i, j)^{\text {th }}$ component of the Wboson. This $\mathrm{W}$-boson has a mass $\left|a_{i}-a_{j}\right|$, while the stretched string has a mass given by string tension $\ell_{s t}{ }^{-2}$ times the length. Thus, the length of the string must be $\ell_{s t}{ }^{2}\left|a_{i}-a_{j}\right|$.

Let us next look at the D4 branes which are sliding between D6 branes and are separated in the $\vec{w}$ direction. It is not obvious with what to identify the states created by strings stretched between them and, therefore, the above argument does not apply to measure the length of the separation in the $\vec{w}$ direction. However, we can compactify on a circle and T-dualize to go to Type IIB theory, and apply the S-duality. Then, the NS 5-branes become D5 branes, D6 branes become NS 5-branes and the D4 branes sliding between D6 branes become D3 branes sliding between NS 5-branes, and we can identify the states created by the string stretched between such D3 branes as the Wbosons in the effective three dimensional gauge theory. This three-dimensional theory is the mirror of the theory obtained by compactification on the circle of the original fourdimensional theory, and the Higgs branch of the original theory is given by the quantum 
Coulomb branch of this mirror theory. Since we know how the moduli parameters are mapped under the mirror symmetry, we can measure the length of the separation in the $\vec{w}$ direction by expressing the same length in terms of the parameters of the mirror gauge theory.

Let $R_{\mathrm{A}}$ be the radius of the compactification circle. After T-duality and S-duality transformations, we obtain Type IIB theory with the string tension $\widetilde{\ell_{s t}}{ }^{-2}=\left(g_{\mathrm{st}} \ell_{s t}{ }^{3} / R_{\mathrm{A}}\right)^{-2}$. The squark $Q_{3}$ of the compactified theory is related to the squark $Q$ of the four-dimensional theory by $Q_{3}=\sqrt{R_{\mathrm{A}}} Q$ and, under the mirror symmetry, the bilinear $\left|Q_{3}\right|^{2}$ is mapped to the scalar component $\widetilde{\phi}$ of the vector multiplet of the mirror. Therefore the length of the separation $\Delta \widetilde{\phi}=\Delta\left|Q_{3}\right|^{2}=R_{\mathrm{A}} \Delta|Q|^{2}=R_{\mathrm{A}} \Delta w$ is given by

$$
\begin{aligned}
\widetilde{\ell}_{s t}^{2}|\Delta \widetilde{\Phi}| & =g_{\mathrm{st}} \ell_{s t}{ }^{3} / R_{\mathrm{A}} \cdot R_{\mathrm{A}}|\mathrm{d} \vec{w}| \\
& =\ell_{11}{ }^{3}|\mathrm{~d} \vec{w}| .
\end{aligned}
$$

There is another test of the metric (A.1) by comparing the brane computation with the field theory result concerning the domain wall of $N=1$ super-Yang-Mills theory with gauge group $S U(n)$. Since the domain wall is a BPS object, we expect that the brane computation will correctly reproduce its tension (For BPS mass formula, see [33, 34, 30] for $N=2$ theory in four dimensions and [58] for $N=2$ theory in two dimensions). In [9, 10], the brane configuration corresponding to the $N=1$ super-YM theory is constructed by rotating the configuration for the $N=2$ super-YM theory. The configuration for the theory with the dynamical scale $\Lambda$ is given by

$$
v w=\Lambda^{3}, \quad t=w^{n}
$$

The domain wall in the brane theory $[10,59]$ is given by a configuration of the fivebrane which varies in the $x^{3}$ direction in such a way that it approaches the configuration (A.3) as $x^{3} \rightarrow-\infty$ while as $x^{3} \rightarrow+\infty$ it approaches the configuration (A.3) with $\Lambda^{3}$ being replaced by $\Lambda^{3} \mathrm{e}^{2 \pi i / n}$. The tension is proportional to the regularized volume of the nontrivial part of the configuration, and is given by the integration of the holomorphic three form times the fivebrane tension $\ell_{11}{ }^{-6}$. The holomorphic three form associated with the metric (A.1) is given by

$$
\Omega=\ell_{s t}{ }^{2} \mathrm{~d} v \wedge \ell_{11}^{3} \mathrm{~d} w \wedge R \frac{\mathrm{d} t}{t}=\ell_{11}{ }^{6} \mathrm{~d} v \wedge \mathrm{d} w \wedge \frac{\mathrm{d} t}{t} .
$$

The computation by [10] leads to the tension formula

$$
\frac{1}{\ell_{11}{ }^{6}}\left|\int \Omega\right|=4 \pi n\left|\Lambda^{3}\left(1-\mathrm{e}^{2 \pi i / n}\right)\right|,
$$


which agrees with the field theory result [60]. ${ }^{1}$

\section{B The Taub-NUT Space}

In the presence of D6 branes at points in the 4,5,6 directions the space-time metric is obtained by replacing $\left|\ell_{s t}{ }^{2} \mathrm{~d} v\right|^{2}+|R \mathrm{~d} t / t|^{2}$ in (A.1) by the metric of the multi-Taub-NUT space. Here we collect some useful facts and formulae about this space (see $[61,62]$ ).

If the D6 branes are at $\vec{x}=\left(x^{4}, x^{5}, x^{6}\right)=\vec{x}_{i}, i=1, \ldots, N_{f}$, the corresponding TaubNUT metric is given by

$$
\mathrm{d} s_{\mathrm{TN}}^{2}=U \mathrm{~d} \vec{x}^{2}+R^{2} U^{-1}\left(\mathrm{~d} x^{10}+\vec{\omega} \cdot \mathrm{d} \vec{x}\right)^{2}
$$

where

$$
U=1+\sum_{i=1}^{N_{f}} \frac{R}{2\left|\vec{x}-\vec{x}_{i}\right|}, \quad \operatorname{curl} \vec{\omega}=\frac{2}{R} \operatorname{grad} U .
$$

Since $x^{10}$ has period $2 \pi$, it is a circle bundle over the $\vec{x}$ space at large $|\vec{x}|$ whose asymptotic radius is $R$. The equation (B.2) shows that $\vec{\omega}$ has magnetic charge $N_{f}$ and hence the circle bundle has first Chern class $N_{f}$ on a two sphere at a fixed large $|\vec{x}|$.

This is a hyper-Kähler space. Among the family of complex structures parametrized by a two-sphere, we choose the one such that $x^{4}+i x^{5}$ is a complex coordinate. For reasons explained in Appendix A, we identify this with $\ell_{s t}{ }^{2} v$ :

$$
v=\ell_{s t}^{-2}\left(x^{4}+i x^{5}\right)
$$

Another complex coordinate has a more involved expression:

$$
y=\mathrm{e}^{-\left(x^{6} / R+i x^{10}\right)} \prod_{i=1}^{N_{f}} \sqrt{\left|\vec{x}-\vec{x}_{i}\right|-\left(x^{6}-x_{i}^{6}\right)} \cdot \text { const } .
$$

In terms of these coordinates, the metric (B.1) is expressed as

$$
\mathrm{d} s_{\mathrm{TN}}^{2}=U\left|\ell_{s t}^{2} \mathrm{~d} v\right|^{2}+R^{2} U^{-1}|\mathrm{~d} y / y-\delta \mathrm{d} v|^{2}
$$

where

$$
\delta=\frac{1}{2} \sum_{i=1}^{N_{f}} \frac{1}{v+m_{i}}\left(1+\frac{x^{6}-x_{i}^{6}}{\left|\vec{x}-\vec{x}_{i}\right|}\right),
$$

\footnotetext{
${ }^{1}$ This refers to the computation in section 5 of [60] of the tension of the domain wall separating two chirally asymmetric vacua of the theory with massive quark multiplets where there is a good description of the low energy theory.
} 
in which $-m_{i}$ are the locations of $\mathrm{D} 6$ branes in the $v$ directions, that is, $m_{i}=-\ell_{s t}{ }^{-2}\left(x_{i}^{4}+\right.$ $\left.i x_{i}^{5}\right)$. If we introduce an another coordinate

$$
x=\mathrm{e}^{x^{6} / R+i x^{10}} \prod_{i=1}^{N_{f}} \sqrt{\left|\vec{x}-\vec{x}_{i}\right|+\left(x^{6}-x_{i}^{6}\right)} \frac{v+m_{i}}{\left|v+m_{i}\right|} \cdot \text { const }
$$

we find the equation

$$
x y=\mathrm{const} \cdot \prod\left(v+m_{i}\right) .
$$

If all $m_{i}$ are the same, it appears from this that there is a singularity at $x=y=v+m=0$, but it is actually smooth as long as $x_{i}^{6}$ are distinct.. This corresponds to the resolution of the $A_{N_{f}-1}$ singularity as described e.g. in $[52,9]$.

Since it is a Ricci flat Kähler manifold with respect to the chosen complex structure, there is a nowhere vanishing holomorphic two-form $\Omega$ such that the square of the Kähler form $\omega$ is given by $\omega^{2}=\Omega \wedge \bar{\Omega}$. It is

$$
\Omega=\ell_{11}^{3} \mathrm{~d} v \wedge \frac{\mathrm{d} y}{y}
$$

The coefficient $\ell_{11}{ }^{3}$ is because of the relation $\ell_{s t}{ }^{2} \cdot R=\ell_{11}{ }^{3}$. That this is nowhere vanishing is obvious in the case where $m_{i}$ are distinct and (B.8) needs no resolution. When $m_{i}$ are coincident (e.g. all zero), that is most easily seen by expressing this in each patch of the resolved surface. In the description of [9], $\Omega=\mathrm{d} x_{i} \wedge \mathrm{d} y_{i}$ up to a constant in the $i^{\text {th }}$ patch coordinatized by $\left(x_{i}, y_{i}\right)$. 


\section{References}

[1] S. Kachru and C. Vafa, "Exact Results for $\mathrm{N}=2$ Compactifications of Heterotic Strings," hep-th/9505105.

[2] S. Kachru, A. Klemm, W. Lerche, P. Mayr and C. Vafa "Nonperturbative Results on the Point Particle Limit of N=2 Heterotic String Compactifications," hep-th/9508155.

[3] A. Klemm, W. Lerche, P. Mayr, C. Vafa and N. Warner, "Self-Dual Strings and N=2 Supersymmetric Field Theory," hep-th/9604034.

[4] A. Hanany and E. Witten, "Type IIB Superstrings, BPS Monopoles, And ThreeDimensional Gauge Dynamics," hep-th/9611230.

[5] E. Witten, "Solutions of Four-Dimensional Field Theories via $M$ Theory," hep-th/9703166.

[6] N. Seiberg and E. Witten, "Monopole Condensation, And Confinement In $N=2$ Supersymmetric Yang-Mills Theory," hep-th/9407087; " Monopoles, Duality and Chiral Symmetry Breaking in $N=2$ Supersymmetric QCD," hep-th/9408099.

[7] S. Elitzur, A. Giveon and D. Kutasov, "Branes and $N=1$ Duality in String Theory," hep-th/9702014.

[8] S. Elitzur, A. Giveon, D. Kutasov, E. Rabinovici and A. Schwimmer, "Brane Dynamics and $N=1$ Supersymmetric Gauge Theory," hep-th/9704104.

[9] K. Hori, H. Ooguri, Y. Oz, "Strong Coupling Dynamics of Four-Dimensional N=1 Gauge Theories from M Theory Fivebrane," hep-th/9706082.

[10] E. Witten, "Branes And The Dynamics Of QCD", hep-th/9706109.

[11] A. Brandhuber, N. Itzhaki, V. Kaplunovsky, J. Sonnenschein and S. Yankielowicz, "Comments on the M Theory Approach to $N=1$ SQCD and Brane Dynamics," hep-th/9706127.

[12] M. Schmaltz and R. Sundrum, " $N=1$ Field Theory Duality from M-theory," hepth/9708015.

[13] J. de Boer and Y. Oz, "Monopole Condensation and Confining Phase of $N=1$ Gauge Theories Via M Theory Fivebrane," hep-th/9708044.

[14] C. Csaki and W. Skiba, "Duality in Sp and SO Gauge Groups from M Theory," hepth/9708082.

[15] C. Ahn, K. Oh and R. Tatar, " $S p\left(N_{c}\right)$ Gauge Theories and M Theory Fivebrane," hepth/9708127; "M Theory Fivebrane Interpretation for Strong Coupling Dynamics of $S O\left(N_{c}\right)$ Gauge Theories," hep-th/9709096.

[16] J. Lykken, E. Poppitz and S. P. Trivedi, " Chiral Gauge Theories from D-Branes," hepth/9708134. 
[17] A. Giveon and O. Pelc," $M$ Theory, Type IIA String and $4 D N=1$ SUSY $S U\left(N_{L}\right) X S U\left(N_{R}\right)$ Gauge Theory," hep-th/9708168.

[18] H. Ooguri, "M Theory Fivebrane and SQCD," hep-th/9709211.

[19] J.L.F. Barbon, A. Pasquinucci, "Softly Broken MQCD and the Theta Angle," hepth/9711030.

[20] A. Fayyazuddin, M. Spalinski, "Extended Objects in MQCD," hep-th/9711083.

[21] I. Bandos, K. Lechner, A. Nurmagambetov, P. Pasti, D. Sorokin and M. Tonin, "Covariant Action for the Super Five-Brane of M-Theory," hep-th/9701149.

[22] M. Aganagic, J. Park, C. Popescu and J.H. Schwarz, "World-Volume Action of the Mtheory Five-Brane," hep-th/9701166.

[23] P.S. Howe, E. Sezgin and P.C. West, 'Covariant Field Equations of the M-theory FiveBrane," hep-th/9702008.

[24] E. Witten, 'Five-Brane Effective Action in $M$-Theory', hep-th/9610234.

[25] R. Güven, 'Black $p$-Brane Solutions of $D=11$ Supergravity Theory', Phys. Lett. B276 (1992) 49.

[26] S. Klemm, W. Lerche, S. Theisen and S. Yankielowicz, "Simple Singularities and N=2 Supersymmetric Yang-Mills Theory," hep-th/9411048.

[27] P. Argyres and A. Faraggi, " The Vacuum Structure and Spectrum of N=2 Supersymmetric SU(N) Gauge Theory," hep-th/9411057.

[28] A. Hanany and Y. Oz, "On the Quantum Moduli Space of Vacua of $N=2$ Supersymmetric $S U\left(N_{c}\right)$ Gauge Theories," hep-th/9505075.

[29] P. C. Argyres, M. R. Plesser and A. D. Shapere, "The Coulomb Phase of N=2 Supersymmetric QCD," hep-th/9505100.

[30] A. Mikhailov, "BPS States and Minimal Surfaces", hep-th/9708068.

[31] P.S. Howe, N.D. Lambert and P.C. West, 'Classical M-Fivebrane Dynamics and Quantum $N=2$ Yang-Mills', hep-th/9710034.

[32] E. Verlinde, "Global Aspects of Electric-Magnetic Duality," hep-th/9506011.

[33] A. Fayyazuddin and M. Spaliński, "The Seiberg-Witten differential from $M$ theory ", hepth/9706087.

[34] M. Henningson, P. Yi, "Four-dimensional BPS-spectra via M-theory", hep-th/9707251.

[35] S.V. Ketov, 'On the Exact Solutions to Quantum $N=2$ Gauge Theories', hep-th/9710085.

[36] M. Henningson, 'Extended Superspace, Higher Derivatives and $S L(2, Z)$ Duality', hepth/9507135. 
[37] M. Matone, 'Modular Invariance and Structure of the Exact Wilsonian Action of $N=2$ SYM', hep-th/9610204.

[38] A. Yung, 'Higher Derivative Terms in the Effective Action of $N=2$ SUSY QCD from Instantons', hep-th/9705181.

[39] S.V. Ketov, 'On the Next-to-Leading-Order Correction to the Effective Action in $N=2$ Gauge Theories', hep-th/9706079.

[40] B. de Wit, M.T. Grisaru and M. Roček, 'Nonholomorphic Corrections to the One Loop $N=2$ Super Yang-Mills Action', hep-th/9601115.

[41] U. Lindström, F. Gonzalez-Rey, M. Roček and R. von Unge, 'On $N=2$ Low Energy Effective Actions', hep-th/9607089.

[42] D. Bellisai, F. Fucito, M. Matone and G. Travaglini, 'Non-Holomorphic Terms in $N=2$ SUSY Wilsonian Actions and RG Equation', hep-th/9706099.

[43] I.L. Buchbinder, S.M. Kuzenko, B.A. Ovrut "On the D = 4, N = 2 Non-Renormalization Theorem", hep-th/9710142

[44] P.S. Howe, N.D. Lambert and P.C. West, 'The Self-Dual String Soliton', hep-th/9709014.

[45] M. Dine, N. Seiberg, 'Comments on Higher Derivative Operators in Some SUSY Field Theories', hep-th/9507057.

[46] A. Kapustin, "The Coulomb branch of $\mathrm{N}=1$ supersymmetric gauge theory with adjoint and fundamental matter," hep-th/9611049.

[47] N. Arkani-Hamed and H. Murayama, "Renormalization Group Invariance of Exact Results in Supersymmetric Gauge Theories," hep-th/9705189.

[48] P.C. Argyres, M.R. Plesser and N. Seiberg. "The Moduli Space of N=2 SUSY QCD and Duality in N=1 SUSY QCD," hep-th/9603042.

[49] N. Seiberg and E. Witten, "Gauge Dynamics And Compactification To Three Dimensions", hep-th/9607163.

[50] K. Intriligator and N. Seiberg, "Mirror Symmetry in Three Dimensional Gauge Theories," hep-th/9607207.

[51] J. de Boer, K. Hori, H. Ooguri, Y. Oz and Z. Yin, "Mirror Symmetry in Three-Dimensional Gauge Theories, $S L(2, Z)$ and D-brane Moduli Space," hep-th/9612131.

[52] K. Hori, H. Ooguri and C. Vafa, "Non-Abelian Conifold Transitions and N=4 Dualities in Three Dimensions," hep-th/9705220.

[53] J. de Boer, K. Hori, H. Ooguri and Y. Oz, "Mirror Symmetry in Three-Dimensional Gauge Theories, Quivers and D-branes," hep-th/9611063. 
[54] G.W. Gibbons, G.T. Horowitz and P.K. Townsend, "Higher Dimensional Resolution of Dilatonic Black Hole Singularities," hep-th/9410073.

[55] B. Greene, D. Morrison and C. Vafa, "A Geometric Realization of Confinement," hepth/9608039.

[56] J. de Boer, K. Hori and Y. Oz, "Dynamics of N=2 Supersymmetric Gauge Theories in Three Dimensions," hep-th/9703100.

[57] J. de Boer, K. Hori, Y. Oz and Z. Yin, "Branes and Mirror Symmetry in $N=2$ Supersymmetric Gauge Theories in Three Dimensions," hep-th/9702154.

[58] A. Hanany, K. Hori, "Branes and $N=2$ Theories in Two Dimensions," hep-th/9707192.

[59] A. Volovich, "Domain Wall in MQCD and Supersymmetric Cycles in Exceptional Holonomy Manifolds," hep-th/9710120.

[60] A. Kovner, M. Shifman, A. Smilga, "Domain Walls in Supersymmetric Yang-Mills Theories", hep-th/9706089.

[61] N.J. Hitchin, "Polygons and gravitons", Math. Proc. Camb. Phil. Soc. 85 (1979) 465.

[62] T. Nakatsu, K. Ohta, T. Yokono and Y. Yoshida, "Higgs Branch of $N=2$ SQCD and $M$ theory Branes", hep-th/9707258. 